\title{
Chemiluminescence Microscopy as a Tool in Biomedical Research
}

\author{
BioTechniques 31:1098-1105 (November 2001)
}

\section{Robbert Créton and Lionel F. Jaffe ${ }^{1}$}

Brown University School of Medicine, Women \& Infants Hospital, Providence, RI, and ${ }^{1}$ Marine Biological Laboratory, Woods Hole, MA, USA cent probes and reporter genes in cells and tissues. Specific methods of photon collection, storage, and analysis have been developed for microscopic imaging of chemiluminescence. Two of these methods are discussed in detail. The first is a method of data storage that allows days of continuous imaging without creating oversized files. The second is a method for calibrating photon imaging microscopes using a low-light standard. Such calibration will be helpful for comparing the performance of various photon imaging systems and for comparing data obtained in different laboratories.

\section{INTRODUCTION}

Chemiluminescence has become a standard tool in biomedical research. Chemiluminescent probes are used for immunoassays, nucleic acid identification, reporter gene assays, measuring enzyme activity, and the detection of ions and small molecules such as $\mathrm{Ca}^{2+}, \mathrm{ATP}, \mathrm{NO}, \mathrm{O}_{2}^{-}$, and $\mathrm{H}_{2} \mathrm{O}_{2}$. Along with the development of new chemiluminescent probes, significant progress has been made in techniques to measure chemiluminescence. Ultra-sensitive photometers or luminometers have become widely available and can be obtained with automatic injectors and microplate readers. In addition, imaging photon detectors have been developed that allow the imaging of chemiluminescence from gels, blots, and microplates. Imaging photon detectors have also been attached to microscopes and allow imaging of chemilumines-
Emission of visible light, without requiring a rise in temperature, is referred to as luminescence. It includes fluorescence, the emission of light following light absorption, and chemiluminescence, the emission of light caused by a chemical reaction. Chemiluminescent assays have virtually no background and are extremely sensitive. This sensitivity has led to the widespread use of chemiluminescent assays in the biomedical sciences $(16,26)$. For example, chemiluminescent indicators are used for immunoassays in microplates, immunolabeling of western blots, and nucleic acid detection in northern and Southern blots. Perhaps one of the most promising applications of chemiluminescence is the direct measurement of various substances at a cellular level.
Cells can be fixed for immunohistochemistry or in situ hybridization using chemiluminescent probes (32). However, it is also possible to use chemiluminescent indicators and reporter genes to study dynamic intracellular processes in living cells (33). Chemiluminescent indicators are well suited for such in vivo studies. Because no exciting light is needed, chemiluminescence can be measured for many days without disturbing the living system. This is in contrast to fluorescence, which can damage living systems by the exciting light, in particular when using light with short wavelengths (ultraviolet or blue light), long exposure times, or exciting light at high intensities. This review provides a brief introduction of chemiluminescent indicators and their applications, provides an overview of general concepts in low-light recording, and describes available techniques in chemiluminescence microscopy.

\section{CHEMILUMINESCENT INDICATORS AND THEIR APPLICATIONS}

A large number of chemiluminescent substances have been identified in nature, and synthetic analogs have been developed to facilitate their use as indicator molecules. The most widely used indicators are shown in Table 1 and are briefly discussed. Special reference is made to the application of these indica- 
Table 1. Examples of Widely Used Bioluminescent and Chemiluminescent Reactions

\begin{tabular}{|c|c|c|c|}
\hline Luminophore & Enzyme & Wavelength & QYa \\
\hline \multirow{2}{*}{\multicolumn{2}{|c|}{$\begin{array}{l}\text { a) Firefly luciferin }+\mathrm{ATP}+\mathrm{O}_{2} \stackrel{\text { luciferase }}{\longrightarrow} \text {-products }+ \text { light } \\
\text { b) coelenterazine }+\mathrm{O}_{2}+\text { apo-aequorin } \longrightarrow \text {-aequorin } \stackrel{\mathrm{Ca}^{2}+}{\longrightarrow} \text {-products }+ \text { light }\end{array}$}} & $563 \mathrm{~nm}$ & $88 \%$ \\
\hline & & $466 \mathrm{~nm}$ & $16 \%$ \\
\hline \multirow{2}{*}{\multicolumn{2}{|c|}{$\begin{array}{l}\text { c) Luminol }+\mathrm{H}_{2} \underset{\mathrm{HRP}}{\longrightarrow} \text { - products + light } \\
\text { d) stabilized dioxetanes } \quad \text { alkaline phosphatase/ } \beta \text {-galactosidase }\end{array}$}} & $428 \mathrm{~nm}$ & $1 \%$ \\
\hline & & variable & variable \\
\hline
\end{tabular}

tors for imaging on a cellular level. For information on other chemiluminescent indicators, we refer the reader to a recent review (17). For additional information on the chemistry of light-emitting reactions, see Reference 22.

\section{Firefly Luciferin}

One of the most efficient chemiluminescent reactions is the oxidation of luciferin in the abdomen of the firefly. This reaction can also be classified as bioluminescence because it involves a chemiluminescent reaction that occurs in a living organism. The oxidation of luciferin by luciferase has a quantum yield of $88 \%$, which means that 88 photons are emitted per 100 molecules of reacting luciferin. The luciferin-luciferase reaction is used in biomedical research for gene expression assays, using the luciferase gene (luc) as a reporter. This approach is particularly useful for the imaging of gene expression in living cells. For example, patterns of luminescence can be visualized in living transgenic fruit flies, which express the luciferase gene under the control of a per promoter (30). When these flies are fed with luciferin-containing food, they luminesce rhythmically for a 24-h period. Moreover, these rhythmic patterns of luminescence can be imaged in single cells of a dissected Malpighian tubule. Similarly, circadian rhythms of luciferase expression have been im aged in a single tobacco seedling (15). The luciferase gene has also been used in mouse embryos to image the onset of paternal gene expression in early development (21). In addition, the luciferase reporter has been used for imaging gene expression in whole mice (12) and tumor progression in rats (31).
The luciferin-luciferase reaction can also be used to detect small amounts of ATP when an excess of luciferin and luciferase are present in the reaction mixture. The ATP assay is used, for example, for bacteriuria testing, cell proliferation assays, and hygiene monitoring within the food industry (19). The ATP detection assay can also be carried out on a cellular level. For example, patterns of ATP have been imaged in cryosections of tumors $(25,39)$. Cells are usually lysed in ATP detection assays. However, it may also be possible to image patterns of ATP in living cells using a relatively stable concentration of luciferase and an excess of luciferin. This possibility was recently explored in live luciferase-expressing HeLa cells attacked by complement (34).

\section{Aequorin}

The photoprotein aequorin emits light in the presence of calcium ions (35). The luminophore in this reaction is coelenterazine, which reacts with molecular-bound oxygen. Aequorin is used as an indicator for measuring patterns of free calcium in living cells (7). The aequorin gene can be locally expressed to allow calcium imaging in specific cell organelles such as the nucleus, endoplasmic reticulum, and mitochondria $(5,33)$. Imaging with aequorin does not disturb the living cell, allowing continuous calcium measurements in a single cell for days.

\section{Luminol}

The chemiluminescent substrate luminol reacts with hydrogen peroxide in the presence of horseradish peroxidase (HRP). HRP-labeled antibodies and probes are used for immunoassays (western and dot blots, and microplates) and nucleic acid identification (Southern and northern blots). The sensitivity of these assays often exceeds the sensitivity of radioactive assays. Moreover, assay sensitivity can be increased using luminol analogues and enhancers (17). On a cellular level, the luminol-HRP reaction can be used for in situ hybridization and immunohistochemistry (32).

Luminol can also be used for the detection of reactive oxygen species such as hydrogen peroxide $\left(\mathrm{H}_{2} \mathrm{O}_{2}\right)$ and the superoxide anion $\left(\mathrm{O}_{2}^{-}\right)$in living cells (10) and can be used to visualize cellular nitric oxide (NO) release (43). Other chemiluminescent indicators such as lucigenin, MCLA, and coelenterazine are more specific for the superoxide anion and have various advantages over luminol $(10,40)$. However, as a general indicator for reactive oxygen species, luminol is often the substrate of choice. One application of luminol is the measurement of reactive oxygen species during the respiratory burst in blood phagocytes. Phagocyte photon emission has been measured in humans undergoing stress or disease, and the capability of phagocytes to emit photons reflects remarkably well the pathophysiological state of the individual (18). Similarly, the respiratory burst during sea urchin fertilization can be monitored in synchronous batches of about 0.2 million eggs by adding luminol to the culture medium (38). Luminol has also been used to measure the release of reactive oxygen species in human sperm $(1,23)$. These measurements revealed differences in reactive oxygen concentrations among sperm from fertile and infertile men $(27,44)$.

Interestingly, cellular reactive oxy- 
gen species generate some luminescence without the addition of luminol. However, the levels of this spontaneous luminescence are orders of magnitude lower than the levels obtained with luminol. A low level of spontaneous luminescence has been measured during the respiratory burst in blood phagocytes (18) and sea urchin eggs $(11,38)$. Other cell types emit spontaneous luminescence as well, reaching levels of 3-20 photons per cell per hour (41). In large populations of cells, this low level of luminescence can be measured and even imaged (14).

\section{Stabilized Dioxetanes}

The recently developed dioxetanes allow the detection of a large number of specific enzymes, including phosphatases, phospholipases, proteases, and hydrolases (17). Stabilized dioxetanes have an enzyme-cleavable protective group. Upon enzymatic removal of the protective group, the dioxetanes decompose while emitting a prolonged glow of light. For example, the stabilized dioxetane AMPPD emits blue light $(470 \mathrm{~nm})$ upon alkaline phosphatase-catalyzed dephosphorylation (6). Chemiluminescent substrates for various enzymes such as alkaline phosphatase, $\beta$-galactosidase, and glucuronidase have been developed. These enzymes have been coupled to antibodies and oligonucleotides that are used for chemiluminescent immunoassays (western and dot blots, and microplates) and nucleic acid detection (Southern and northern blots). In addition, the genes coding for these enzymes can be used in chemiluminescent reporter gene assays (29). Chemiluminescent substrates with different colors and kinetics (glow and flash type) are available, thus allowing the recording of various indicators in a single sample (20). The stabilized dioxetanes can also be used on a cellular level for in situ hybridization, immunohistochemistry (32), and enzyme activity detection (17). Moreover, the stabilized dioxetanes could become powerful tools for imaging enzyme activity and expression of reporter genes in living cells. To this end, stabilized dioxetanes that have high quantum yields in physiological solutions need to be developed.

Table 2. Conversion of Light Units

\begin{tabular}{|c|c|c|}
\hline & Unit & Conversion \\
\hline \multicolumn{3}{|l|}{ Photometric } \\
\hline Luminous flux & lumen (Im) & $1 \mathrm{Im}=1 / 683 \mathrm{~W} 555-\mathrm{nm}$ light \\
\hline Luminous intensity & candela $(\mathrm{cd})$ & $1 \mathrm{~cd}=1 \mathrm{Im} /$ steradiana \\
\hline Illuminance & $\begin{array}{l}\text { lux (Ix) } \\
\text { foot-candle (fc) }\end{array}$ & $\begin{array}{l}1 \mathrm{~lx}=1 \mathrm{Im} / \mathrm{m}^{2} \\
1 \mathrm{fc}=1 \mathrm{Im} / \mathrm{ft}^{2}\end{array}$ \\
\hline \multicolumn{3}{|l|}{ Radiometric } \\
\hline Radiant flux & $\begin{array}{l}\text { W } \\
\text { photons/s }\end{array}$ & $\begin{array}{l}1 \mathrm{~W}=1 \mathrm{~J} / \mathrm{s} \\
1 \text { photon }(550 \mathrm{~nm}) / \mathrm{s}=3.6 \times 10^{-19} \mathrm{~Wb}\end{array}$ \\
\hline $\begin{array}{l}\text { Radiant intensity } \\
\text { Irradiance }\end{array}$ & $\begin{array}{l}\text { W/steradian } \\
\mathrm{W} / \mathrm{m}^{2} \\
\text { photons } /\left(\mathrm{s} \times \mathrm{cm}^{2}\right)\end{array}$ & $\begin{array}{l}1 \mathrm{~W} / \text { steradian }=4 \pi \mathrm{W} / \text { sphere } \\
\text { as above } \\
\text { as above }\end{array}$ \\
\hline
\end{tabular}

\section{MEASURING CHEMI- LUMINESCENCE}

\section{Units of Light}

The SI unit of light is the candela (cd), which is defined as the luminous intensity of monochromatic radiation with a wavelength of $555 \mathrm{~nm}$ and an intensity of 1/683 W/steradian (see Table 2 for the conversions of various units). One candela corresponds to one lu$\mathrm{men} / \mathrm{steradian}$ or to one lux with a detector $1 \mathrm{~m}$ away from the light source. The candela, lumen, and lux are photometric units that describe the brightness of light as perceived by the human eye. The average human eye is more sensitive in the green than it is in the red or blue. Thus, relatively high intensities are needed in the blue and red to get the same perceived brightness. Radiometric units of light do not depend on the sensitivity of the human eye and are expressed in $\mathrm{W} / \mathrm{m}^{2}$ or in photons $/\left(\mathrm{s} \times \mathrm{c} \mathrm{m}^{2}\right)$ when intensities are low.

\section{Light Meters}

Ambient levels of light can be measured with light meters, which are based on a silicon photodiode. Such light meters are, for example, used for measuring room light and laser intensity. They display light intensities in photometric units (lux) or in radiometric units $\left(\mathrm{W} / \mathrm{m}^{2}\right)$. Light meters that display intensities in radiometric units are also referred to as optical power meters or radiometers. The most accurate light meters are calibrated using a standard light source (e.g., from the U.S. National Institute of Standards and Technology) and allow for the measurement of light at various wavelengths.

\section{Luminometers}

Chemiluminescence is typically measured with a photometer or luminometer, which is built around a photomultiplier tube (42). The photomultiplier tube is a vacuum tube containing a photocathode, electron multiplier plates, and an electron-collecting anode. A wide variety of luminometers are currently available and include those with automatic reagent injectors and microplate readers (4). For a list of distributors, see Reference 36. The sensitivity of a photomultiplier tube is usually expressed in noise levels and photocathode quantum efficiency, which is the probability that an incident photon will cause the release of an electron. The quantum efficiency depends on the wavelength of the incident photon [i.e., the quantum efficiency is generally best (about 20\%) in the blue and decreases at higher wavelengths (green and red)]. Photomultiplier tubes with good sensitivity in the red and infrared are available ( $\mathrm{S} 1$ response) but tend to have more dark noise. This dark noise can be reduced by cooling the detector. Many luminome- 
ters are operated in a "photon counting" mode, which means that each large pulse is counted as a single photon, while the smaller noise-related pulses are excluded from the recording. This method im proves the sensitivity of the detector but lowers the level at which the luminometer saturates.

Advances in solid-state technology may eventually lead to the development of a photodiode that will replace the vacuum photomultiplier tube. For example, the recently developed "avalanche photodiode" is an extremely sensitive light detector that has an electron amplification mechanism similar to the photomultiplier tube (42).

Luminometers are generally not calibrated in absolute terms (37). Thus, the level of light is displayed in arbitrary units such as relative light units or counts per second. For most practical purposes, these relative units are good enough because internal standards can be included in the experimental protocol. For example, ATP measurements can be calibrated by adding a few standards in which the ATP concentration is known. However, an absolute calibration is essential for comparing the performance of luminometers in different laboratories. An effective method for calibrating luminometers has been previously described (28): a known amount of luminol is burned up in a luminometer, and the recorded counts are compared to the total number of emitted photons. The total number of photons can be calculated based on the quantum yield $(0.0124)$ of the luminol reaction. In addition, various other standards have been developed that are suitable for day-to-day calibrations $(28,37)$.

\section{IMAGING OF CHEMI- LUMINESCENCE}

Sensitive photon imaging systems have been developed for both astronomers and biologists to look at ultra-low levels of light. Many of these systems are now commercially available (36). Three types of cameras form the basis of these imaging systems: slowscan cooled charge-coupled device (CCD) cameras, intensified CCD cameras, and imaging photon detectors (13).

The slow-scan cooled CCD camera is a solid-state device with a high quantum efficiency, in particular when using a back-illuminated chip (4). Quantum efficiencies can be as high as $80 \%$, meaning that 80 out of 100 incident photons are recorded. However, CCD cameras also have a high level of noise, which limits their sensitivity. For measuring chemiluminescence, this noise needs to be reduced by cooling the detector (reducing the dark counts) and by using slow read-out speeds (reducing the read-out noise).

Intensified CCD cameras have an im age intensifier placed in front of a CCD camera. Silicon intensifiers are available but are generally not sensitive enough for imaging low levels of chemiluminescence. The most sensitive intensifiers are the microchannel plate image intensifiers, which are vacuum tubes similar to the photomultiplier tube. When a photon strikes the detector, an electron is released by the photocathode. The signal is amplified within the microchannel plates and transformed to visible light by a phosphor output window. This light is then recorded with a CCD camera, which can be read out quickly and does not need to be cooled. The most sensitive detectors have multiple layers of microchannel plates (e.g., 2- or 3-stage microchannel plates) and allow for photon counting. Similar to the photon counting mode of the photomultiplier tube, the electrical pulses representing an incident photon are recorded, while the smaller noise-related pulses are excluded. Extremely large pulses caused by radioactivity or cosmic radiation can be excluded from the recording as well. The main strength of microchannel plate-intensified cameras is their low level of noise (typically 10-100 counts per second over the entire detector surface). This low noise makes the intensified camera extremely sensitive and allows for the detection of low levels of chemiluminescence. Microchannel plate intensifiers are particularly useful for measuring $d y-$ namic patterns of chemiluminescence because the detector noise does not increase at a fast recording speed. Quantum efficiencies of intensifiers usually peak in the blue and are much lower than quantum efficiencies of solid-state detectors. We have tested various microchannel plate-intensified cameras and estimate that the peak quantum effi- ciency typically ranges between $1 \%$ and $5 \%$. These estimates are lower than values reported by others. As with photomultiplier tubes, detectors with extended sensitivity in the red are available. These detectors need to be cooled to reduce the level of noise.

Imaging photon detectors (IPD) use a microchannel plate intensifier similar to the intensified CCD cameras described above. The difference is that, instead of a phosphor screen, the IPD uses a resistive anode to collect the amplified electron bundles. The signals from the resistive anode are sent directly to a processing unit and stored in the computer as a list of photon events. Thus, IPD-type cameras do not have a phosphor screen or a CCD camera and lack the noise caused by these components. Our laboratory uses a S20 IPD detector (Photek, East Sussex, UK) that is mounted on an Axiovert 100TV inverted microscope (Carl Zeiss, Thornwood, NY, USA) (7). The detector has three stages of microchannel plates, extended sensitivity in the red (S20 response), and is cooled to reduce detector noise. Based on calibrations with a set of light-emitting diodes (LEDs) and a NIST-traceable optical power meter (PD200; Ophir Optronics, Danvers, MA, USA), we estimate that our imaging system has a quantum efficiency of $3.0 \%$ at $430 \mathrm{~nm}$ (blue), $1.7 \%$ at $565 \mathrm{~nm}$ (green), and $0.8 \%$ at $635 \mathrm{~nm}$ (red). These estimates are lower than values reported by others. However, even at quantum efficiencies between $1 \%$ and $5 \%$, the resistive anode IPD may well be the most sensitive detector currently available because of its extremely low level of noise. The IPD system in our laboratory has a noise level of 2 counts per second in a $100 \times 100$-pixel field $(1$ pixel = approximately 1 microchannel). This corresponds to a noise level of 0.0002 counts/pixel second. For the storage and analysis of the photon data, we use custom-written IPD software obtained from Science Wares (East Falmouth, MA, USA). We have used this imaging system to study calcium patterns during embryonic development via the chemiluminescent aequorins. For example, calcium gradients were found to be associated with dorsal-ventral specification in Drosophila embryos (8) and with brain regionalization 
in zebrafish embryos (9).

When choosing a camera, one should consider the spectral response, quantum efficiency, noise, dynamic range, spatial resolution, and ease of use. However, these factors are not easy to evaluate from published specifications. Thus, in general, it is a good idea to try out a camera using the cells or samples that one is interested in. Other factors to consider are the associated software for data storage and analysis, the lens system that collects the light, the detector cooling system, dry air to prevent cooling-

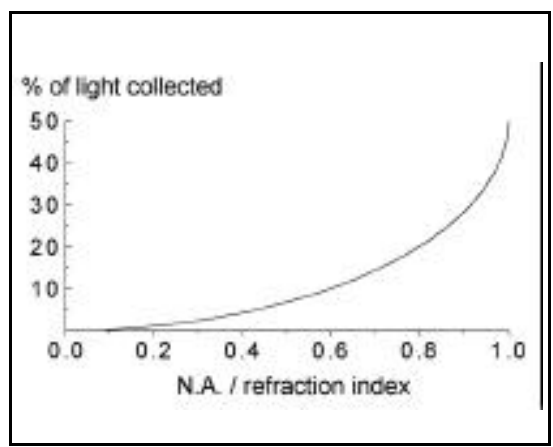

Figure 1. The maximum percentage of light collected depends on the N.A. of the microscope objective. The maximum percentage of light collected was calculated using the angle of acceptance $(\alpha)$, which is the inverse sinus of the N.A. (for dry objectives). The percentage of collected light $=(0.5-0.5 \times \cos \alpha) \times 100 \%$, assum ing that light spreads uniformly in all directions. The actual values of collected light will be slightly lower than these calculated values because of reflectance and absorption of light within the objectives. For immersion objectives, the N.A. needs to be divided by the refraction index of the immersion fluid (1.5 for oil immersion, 1.3 for water immersion, and 1.0 for dry objectives). related condensation, shutters to prevent overexposure, and methods for obtaining bright-field images.

\section{LIGHT COLLECTION ON A PHOTON COUNTING MICROSCOPE}

Photon counting cameras can be attached to microscopes for imaging of chemiluminescence on a cellular level. On a micro-scale, the level of chemiluminescence is often a limiting factor because small objects generally emit a low amount of light (assuming equal concentrations of chemiluminescent reactants). The amount of emitted light is proportional with the volume of a cell and, thus, with the third power of the cell diameter, as long as the cell is transparent (7). For example, upon injection of aequorin, a zebrafish egg with a diameter of $1 \mathrm{~mm}$ emits a thousand times more light than a sea urchin egg with a diameter of $0.1 \mathrm{~mm}$. For opaque tissues, the amount of emitted light is proportional with the surface area and, thus, with the second power of the tissue diameter. Because the amount of light is often limiting, approaches to chemiluminescence microscopy are designed to maximize photon emission and increase the efficiency of the light collection. The most important factor in collection efficiency is the numerical aperture (N.A.) of the objective (Figure 1). A low N.A. objective will collect only a small fraction of the emitted light and will thus decrease the overall sensitivity of the imaging

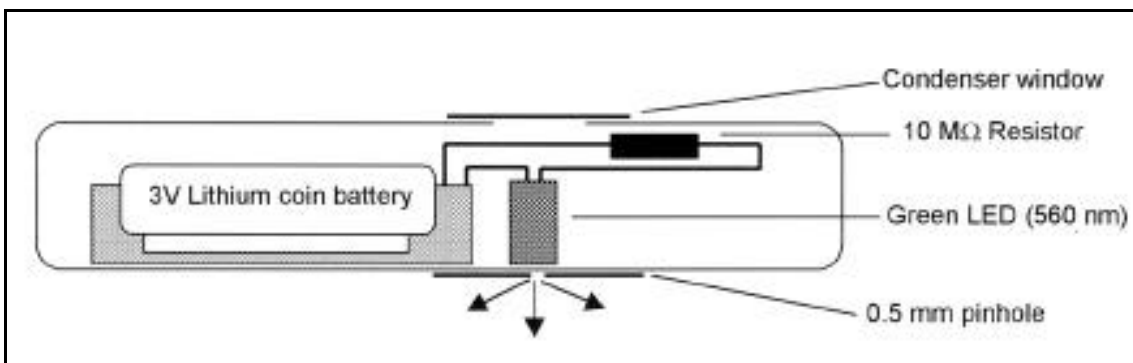

Figure 2. A low-light standard for calibration of photon imaging microscopes. The light source is a green LED, which emits a low level of light through a $0.5-\mathrm{mm}$ pinhole. The standard is battery-powered with a lithium coin battery that has flat discharge characteristics and a long shelf life. The standard does not have an off-switch because the required current is so low that the batteries should last for more than 50 years (about five times longer than the shelf life of the lithium batteries). The condenser window allows condenser light to shine through the LED and pinhole, which is helpful for bringing the pinhole in focus. We estimate that the standards emit about 30000 photons/s through the pinhole. 
system. For example, a dry objective with an N.A. of 0.2 collects only $1 \%$ of the light emitted by a cell or tissue.

\section{IMPROVEMENTS IN CHEMI- LUMINESCENCE MICROSCOPY}

Two specific improvements in chemiluminescence microscopy are discussed in this review. The first is an efficient method of data storage that allows days of continuous imaging without creating oversized files. The second is the development of a low-light standard that can be used to calibrate photon imaging microscopes.

\section{Data Storage}

Imaging data is typically stored as a sequence of images. However, this method of data storage requires a lot of storage space, limiting either the total recording time or the rate of acquisition. For example, an hour recording at one image per second requires approximately one gigabyte (GB) of memory [3600 pictures $\times 0.3$ megabytes $(\mathrm{MB})]$. A more efficient method of data storage is available for photon imaging. Photon data can be stored as a list of photon events in which each photon is labeled by space $(x, y)$ and time coordinates. A single photon event can be stored in 10 bytes. Thus, a continuous $1-\mathrm{h}$ recording at 100 photons/s only requires $3.6 \mathrm{MB}$ of memory $(3600 \mathrm{~s} \times 100$ photons/s $\times$ 10 bytes/photon) instead of a GB. This method has previously been used for IPD-type detectors in which the output is already in the format of a list of photon events (24). However, the same method could be adjusted for storing data obtained with other types of detectors. The potential benefit is that it would allow for days of continuous imaging at a high acquisition rate without creating oversized files.

\section{Low-Light Standard for Calibration of Photon Imaging Microscopes}

Various types of cameras are used to image chemiluminescence, and new solid-state imaging systems may be developed in the near future. It is difficult to compare the performance of these cam eras, various microscopic imaging sys- tems, and results obtained in different laboratories. For such comparison, photon imaging microscopes need to be calibrated. Standard light sources that can be used for calibrating ambient levels of light are available but are generally too big and bright for the calibration of photon imaging systems. An elegant standard for calibrating fluorescence microscopes was previously described (3). This standard consists of a feedback-stabilized LED that emits light through a pinhole. In addition, a dual-wavelength standard was developed for calibrating ratiometric imaging data obtained with fluorescence microscopes (2).

We have recently developed an ultra-low-light standard for photon imaging microscopes by strongly reducing the electrical current through an LED. A $10-\mathrm{M} \Omega$ resistor was placed in series between a green LED (560 $\mathrm{nm})$ and a 3-V lithium battery (Figure 2). A spectral analysis with bandpass filters suggested that the strong reduction in current does not change the color of the emitted light. The average standard gives 13.5 counts/s on our imaging system $(n=8$ and $\mathrm{SEM}=0.8)$ using a PlanApochromat $5 \times$ objective with a N.A. of 0.16 (Carl Zeiss). The geometry of light emission was studied by tilting the standards. Maximum intensities were measured at a tilting angle between 0 and $10^{\circ}$, while the intensity drops to $50 \%$ at a tilting angle of $50^{\circ}$. We estimate that about 30000 photons/s are emitted through the pinhole of the standard. In calculating this value, we assume that the quantum efficiency of the detector is $1.7 \%$ at $560 \mathrm{~nm}$, correct for the percentage of light collected by the objective (1.3\% of a half sphere) and for the geometry of light emission (average intensity is $55 \%$ of maximum). The standards were also investigated using two other microscope objectives. A Fluar $10 \times$ objective with an N.A. of 0.5 (Carl Zeiss) collected 66 counts/s ( $n$ $=8$ and SEM=5), and a Fluar $20 \times$ objective with an N.A. of 0.75 collected 163 counts/s $(n=8$ and SEM $=11)$. These values are somewhat lower than expected, based on the N.A. and geom etry of light emission. Possibly, some light is lost by reflection, which could be significant in high N.A. lenses without the aid of oil immersion. A number of standards were built and will be

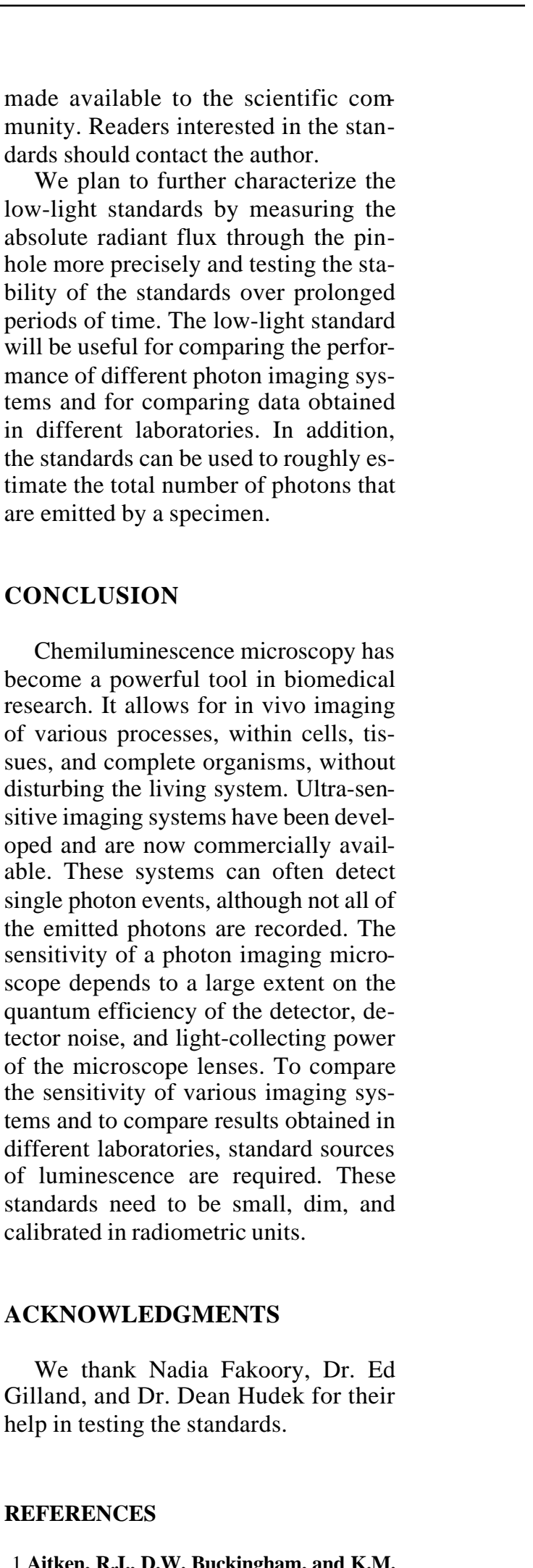

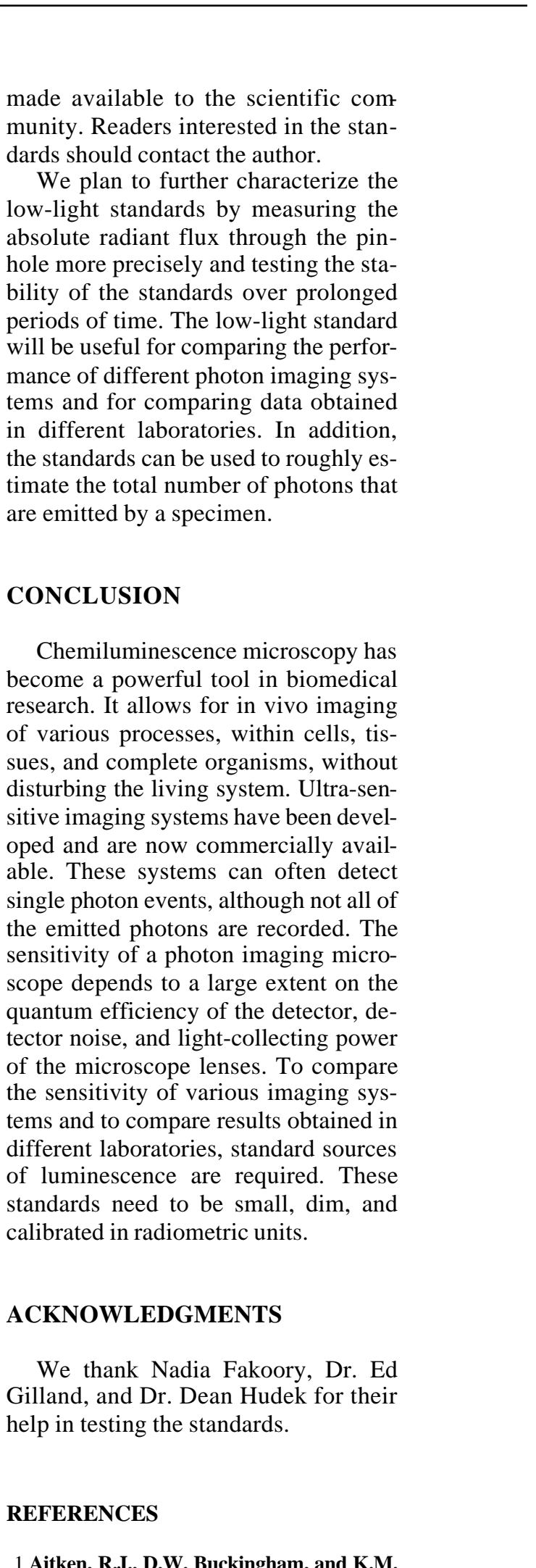

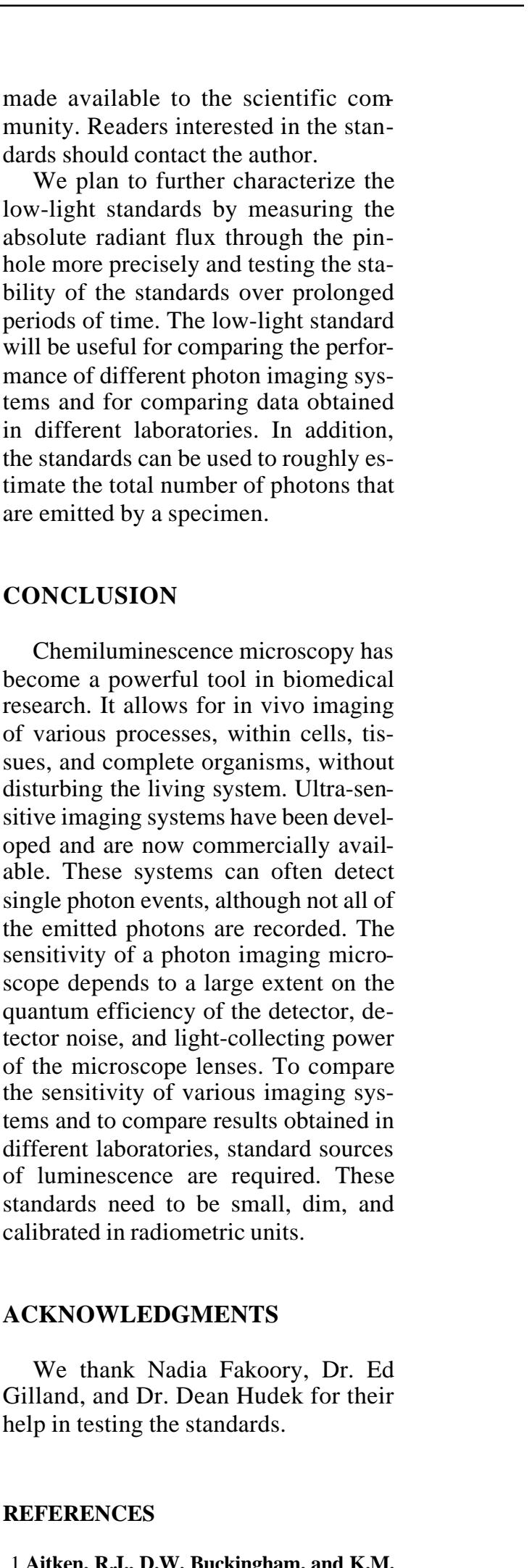

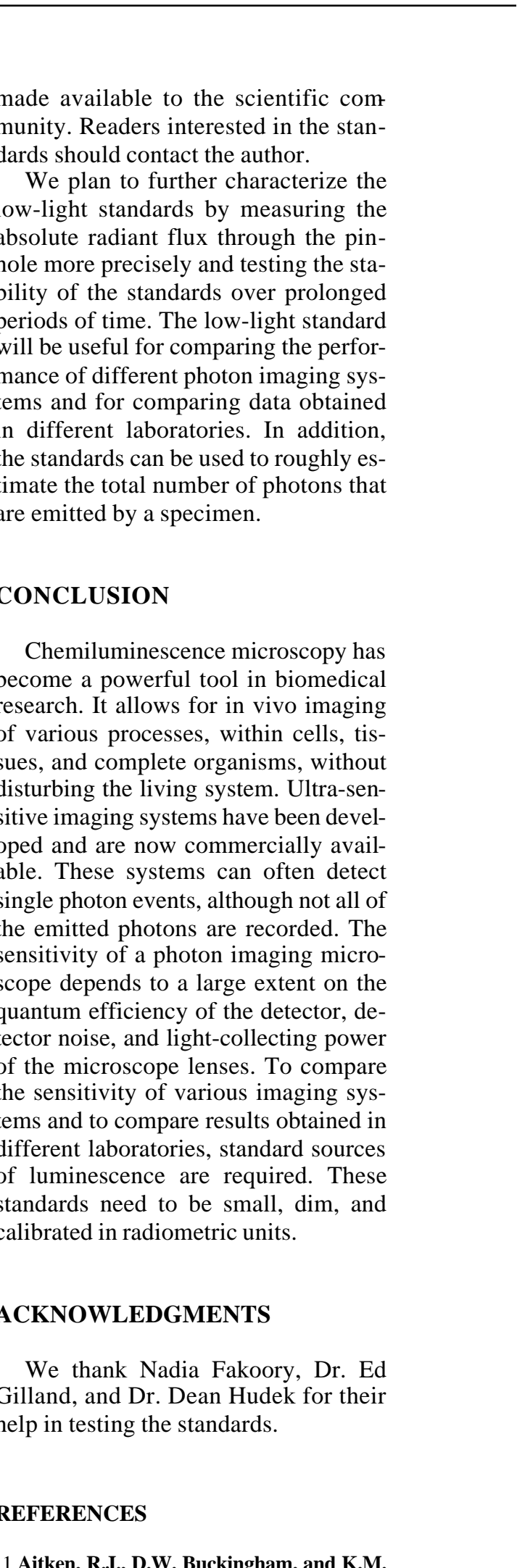

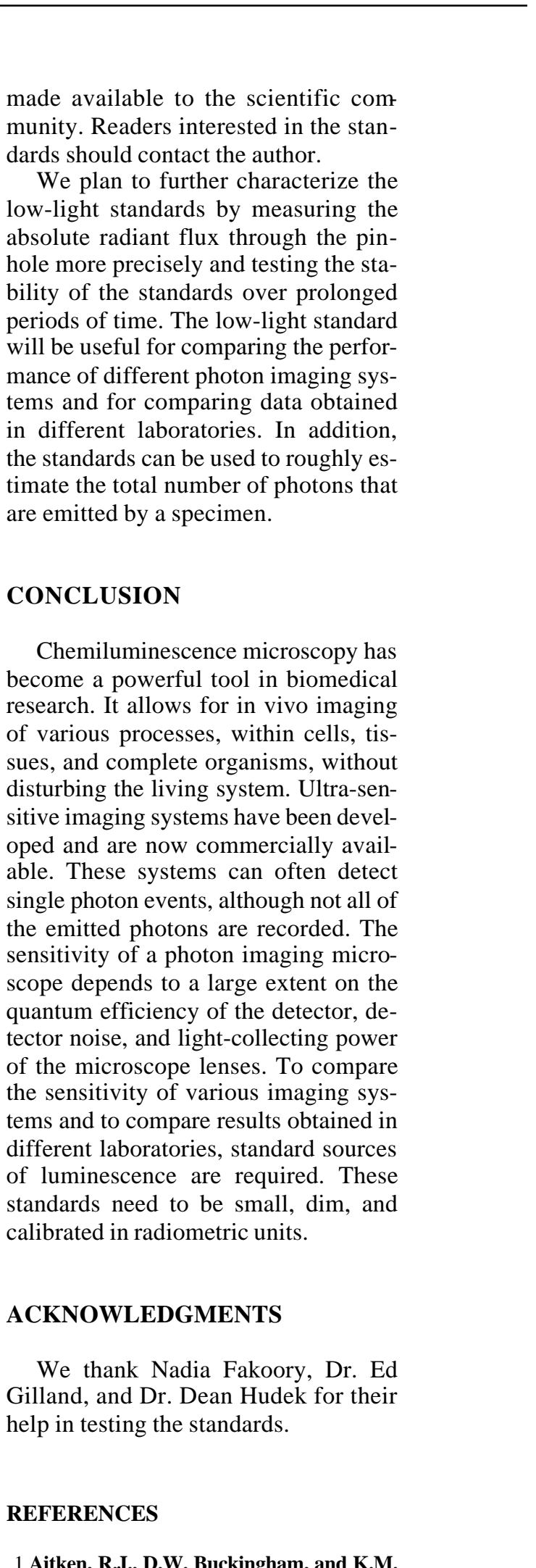

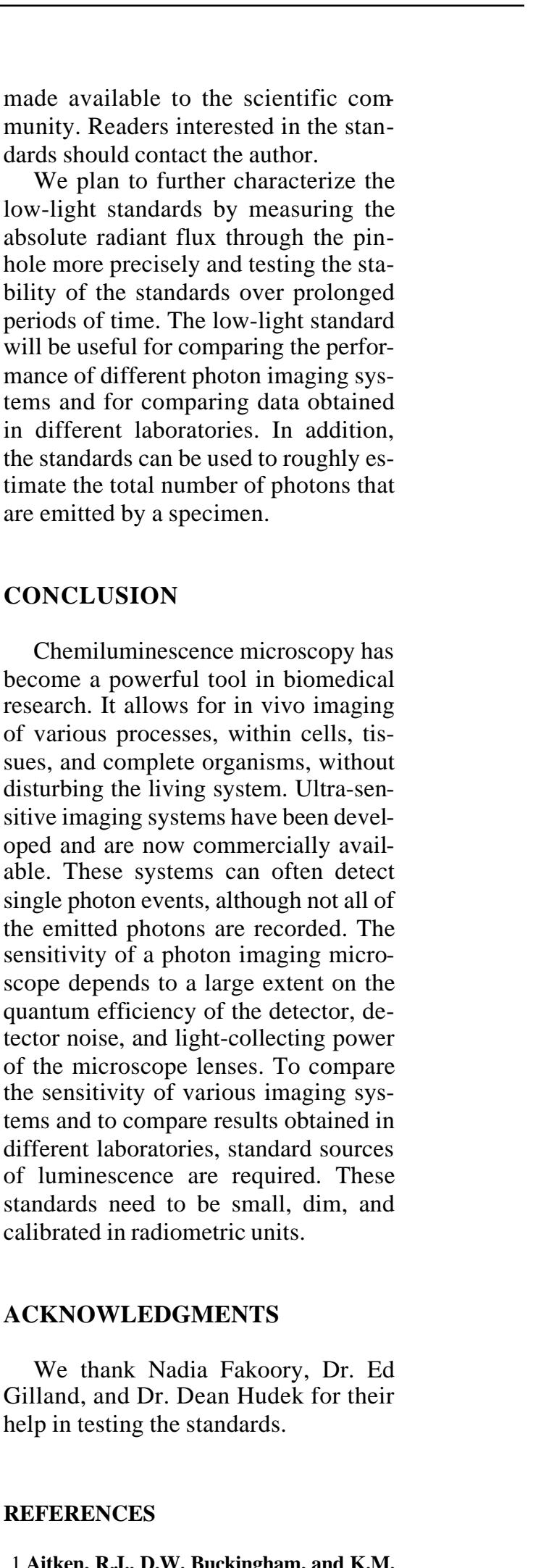

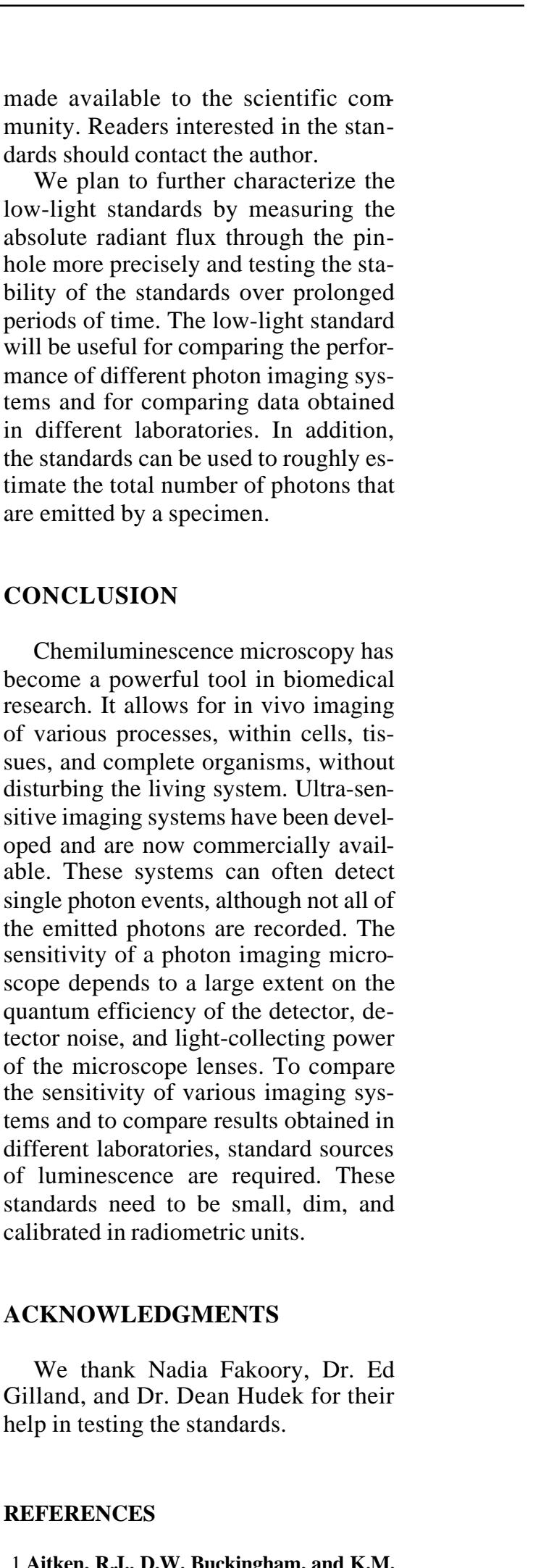

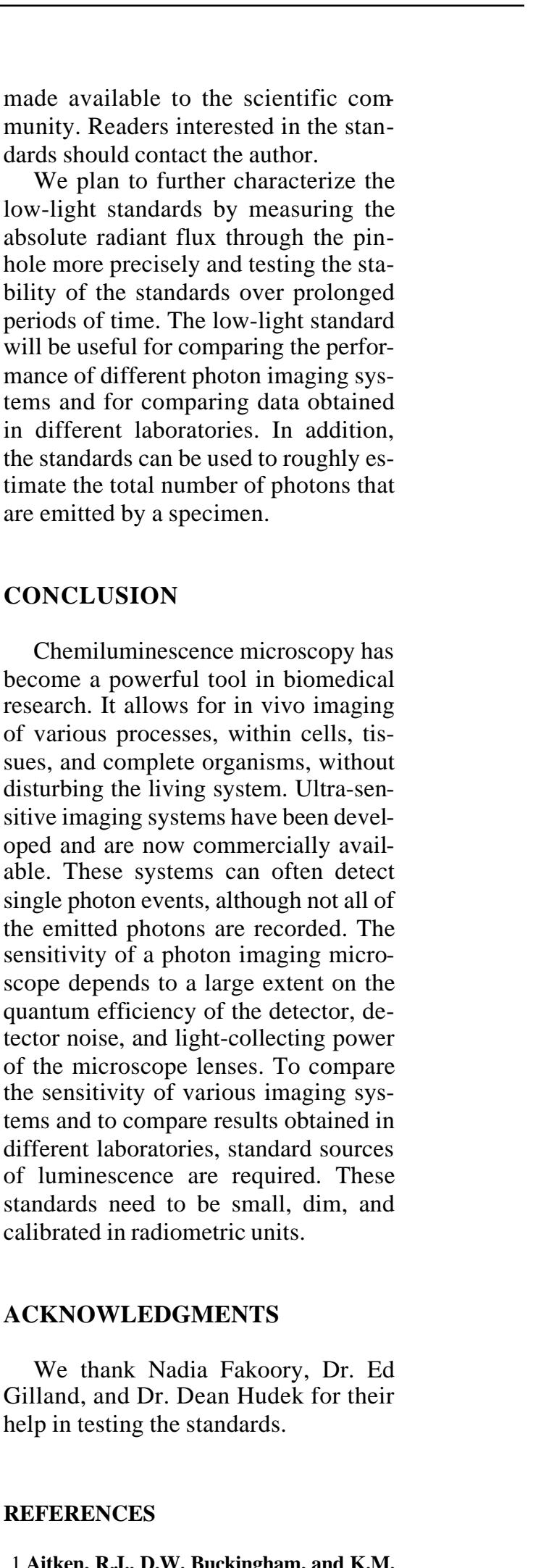

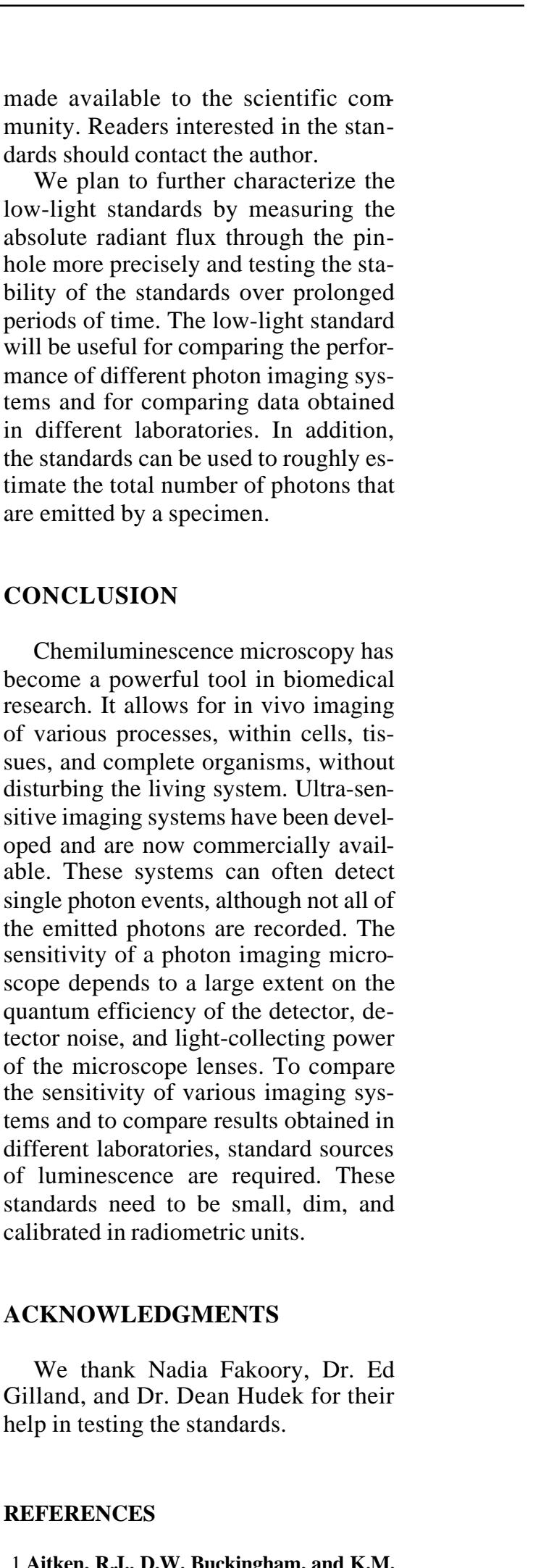

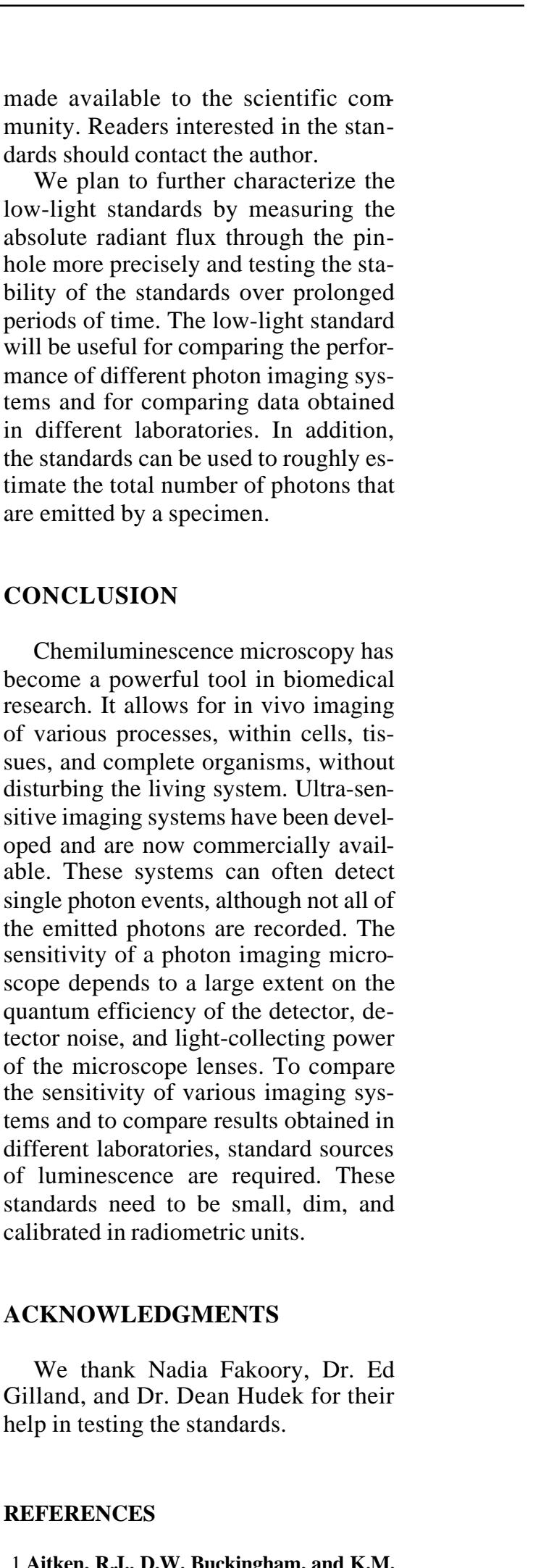

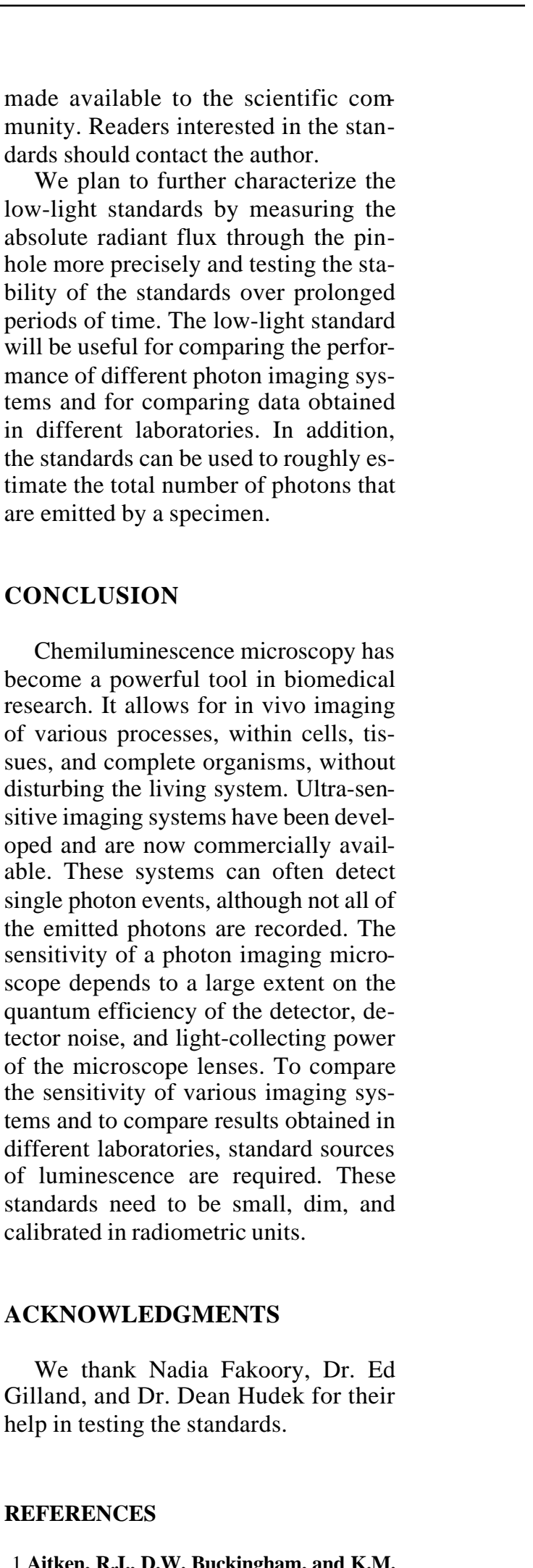

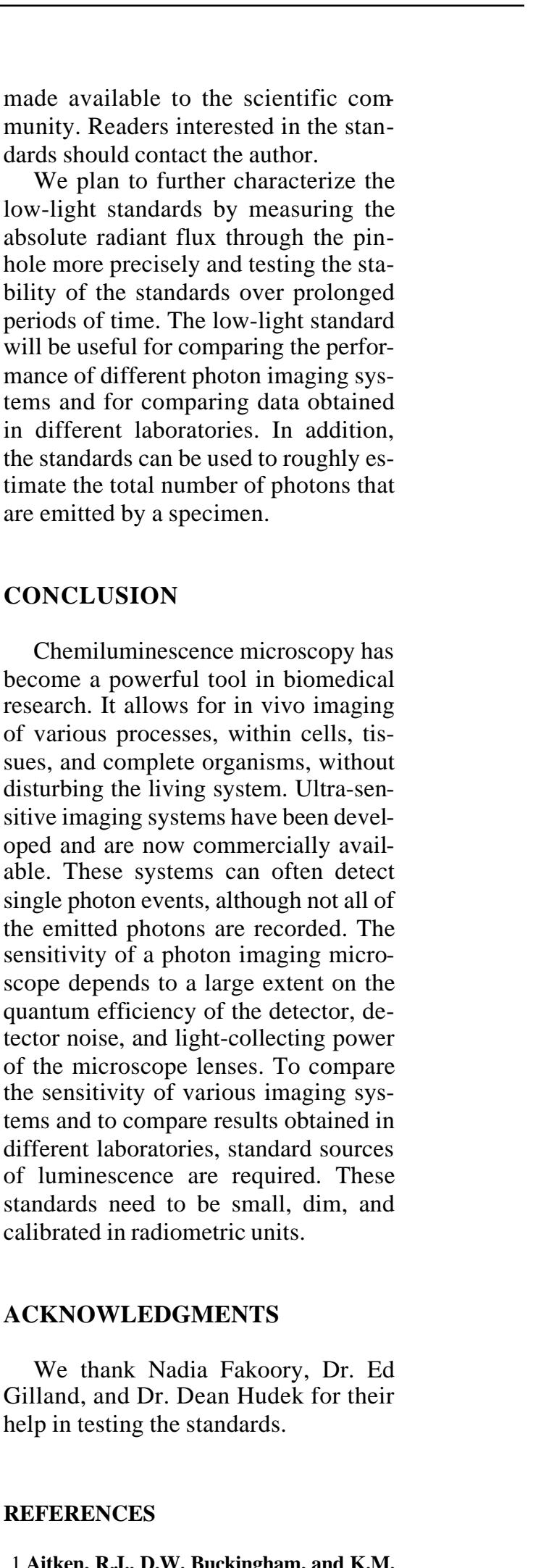

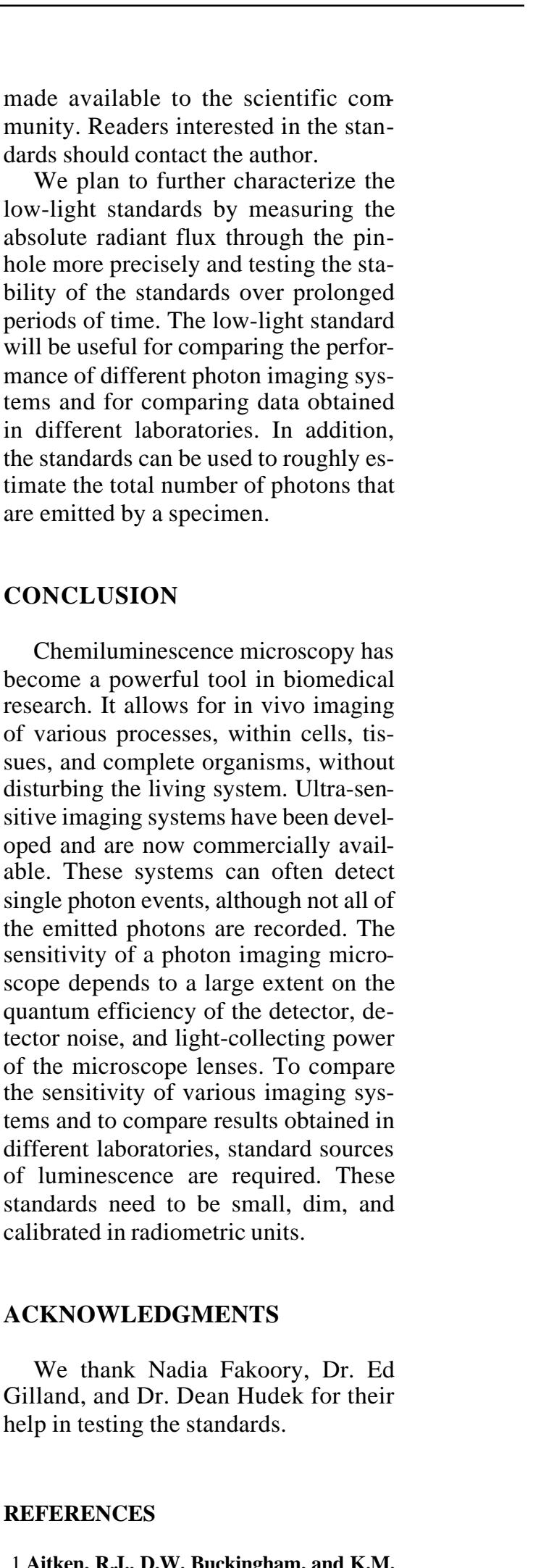

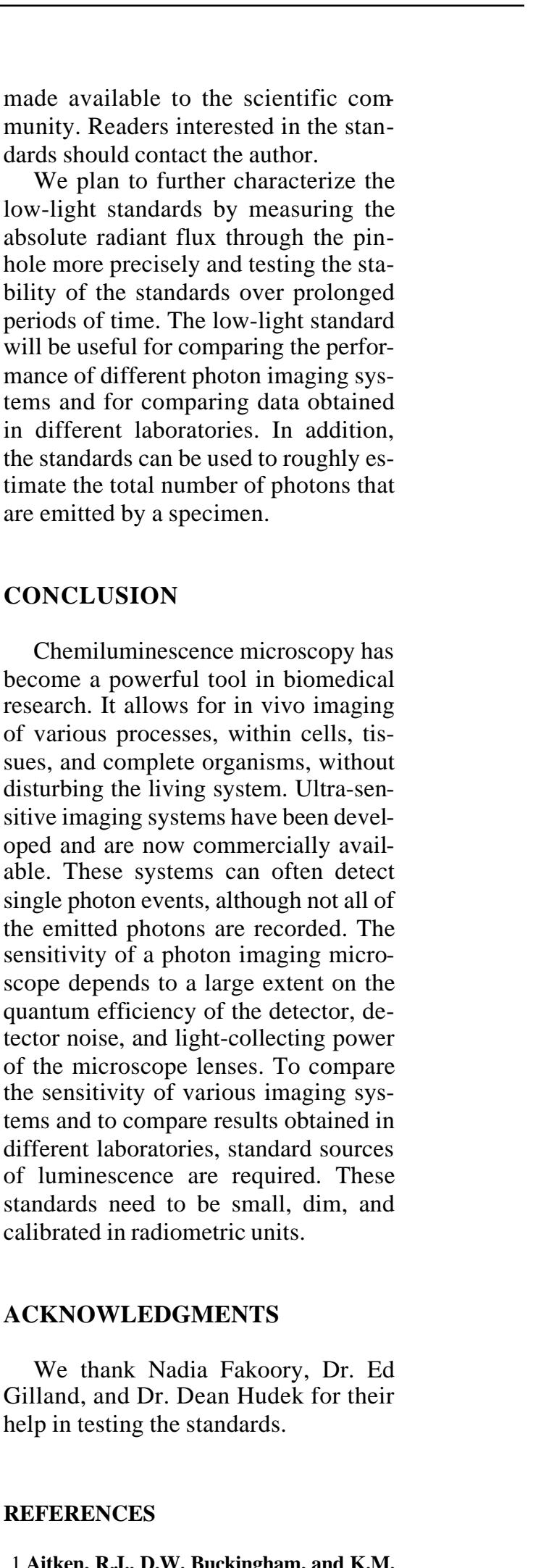

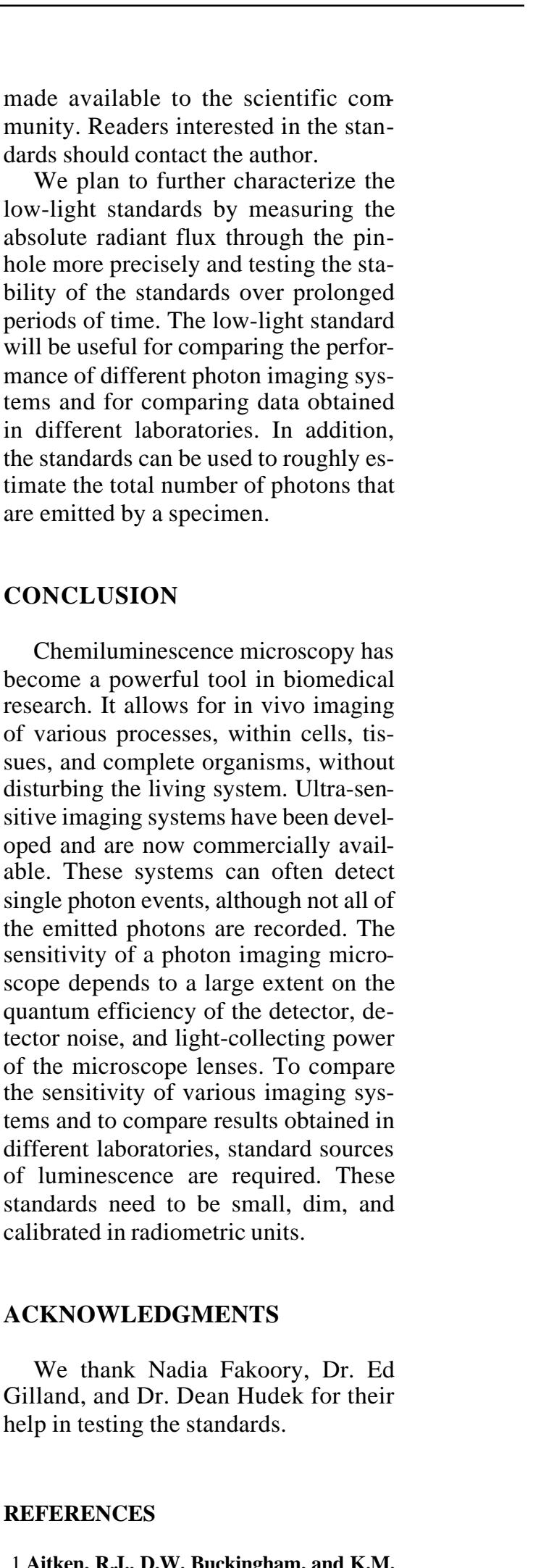

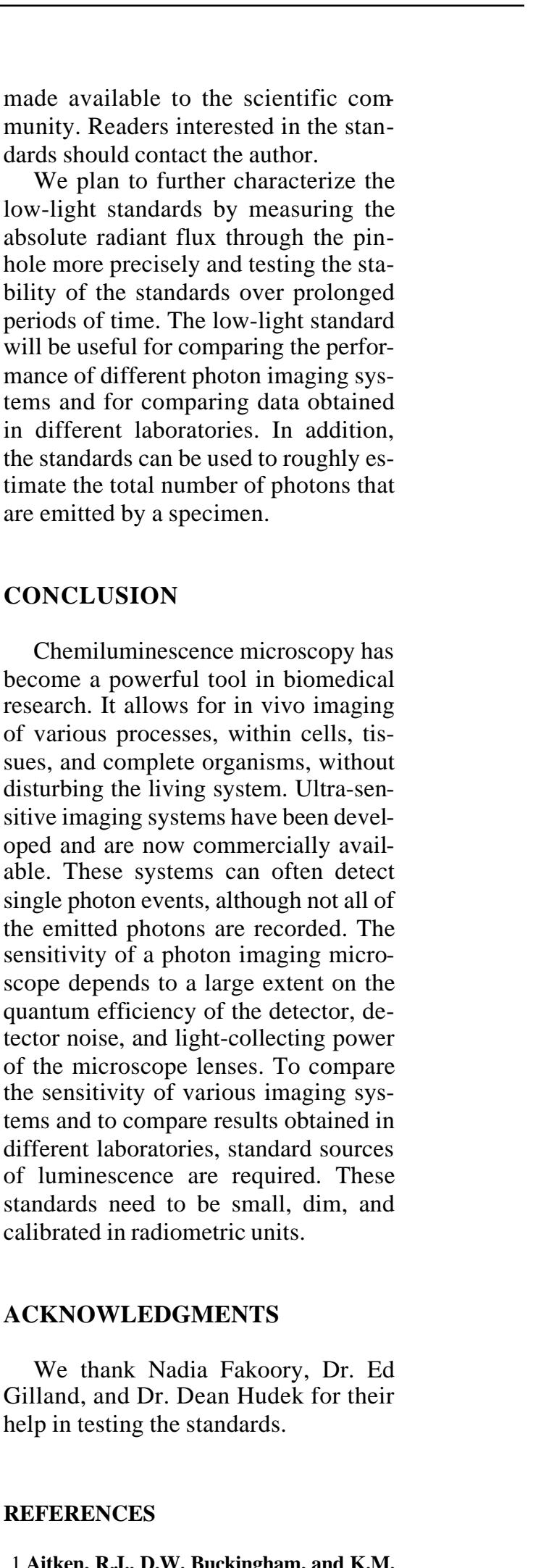

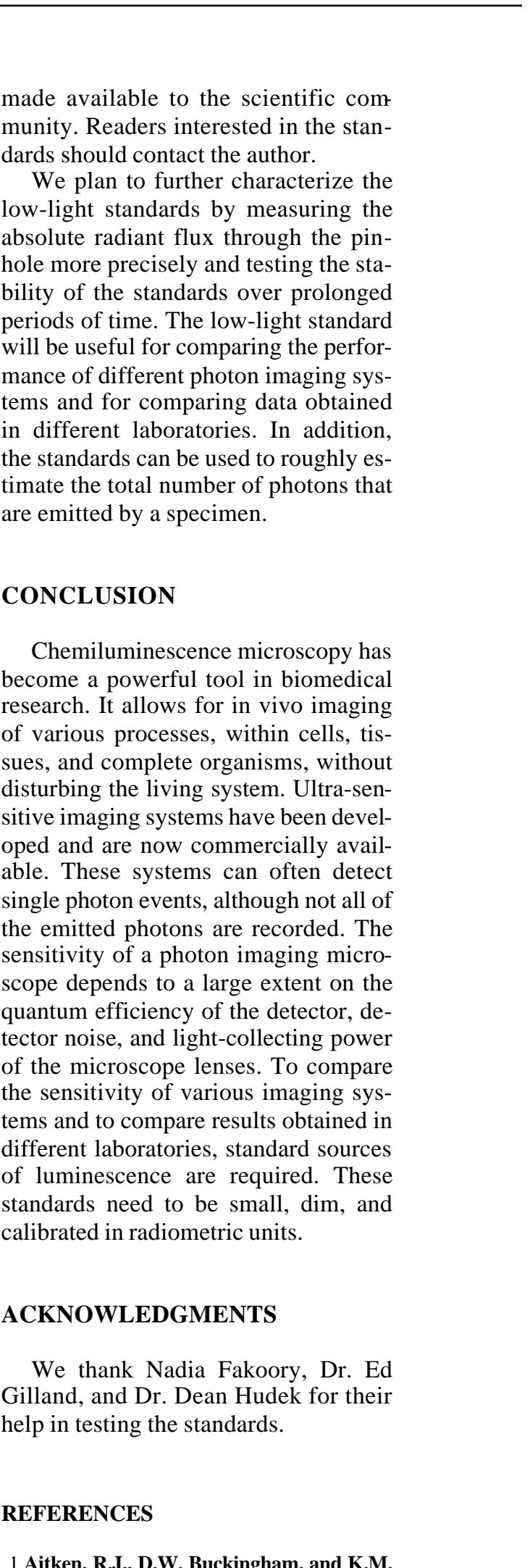

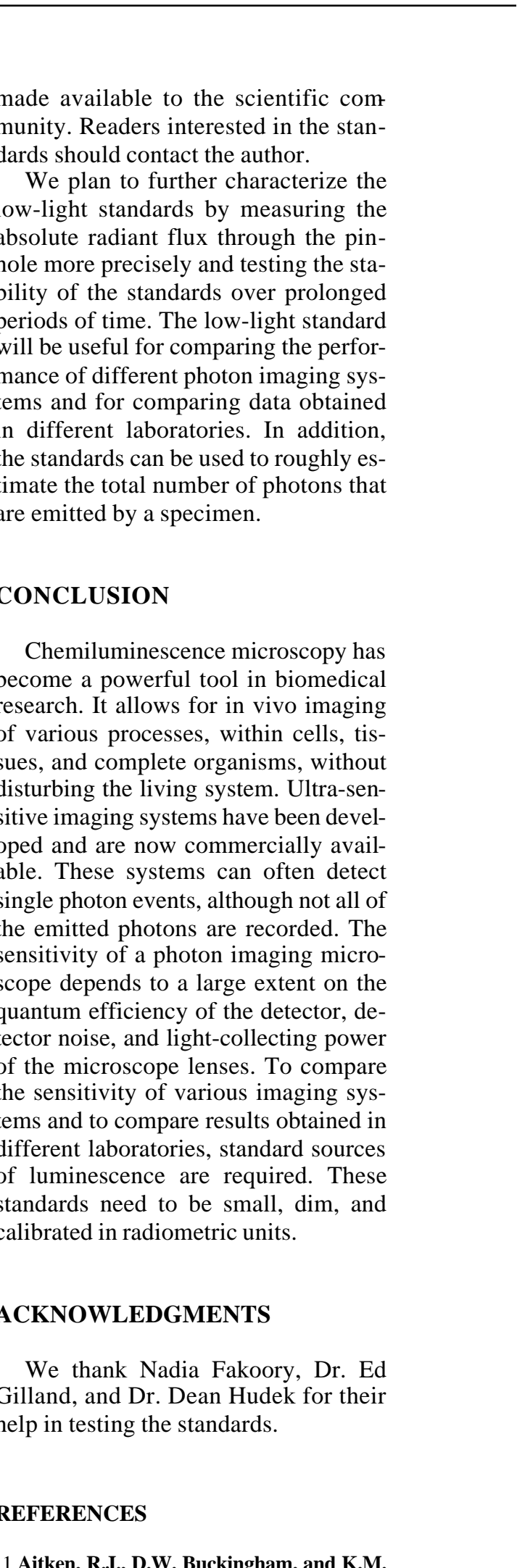

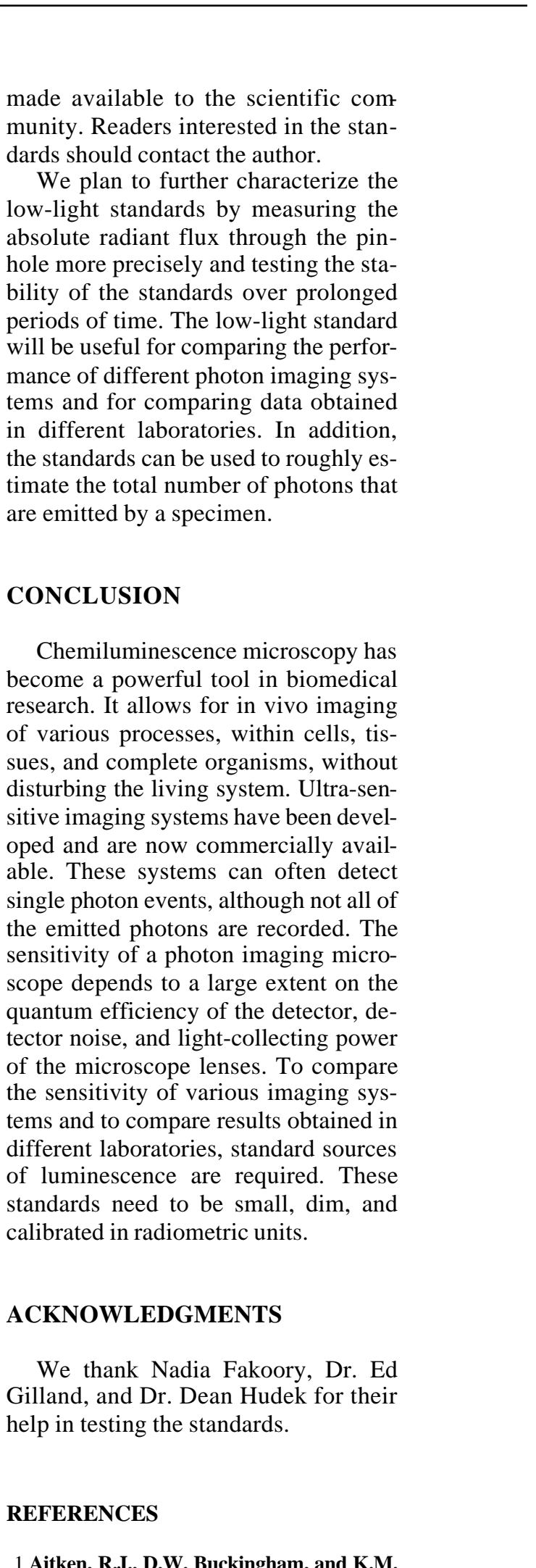

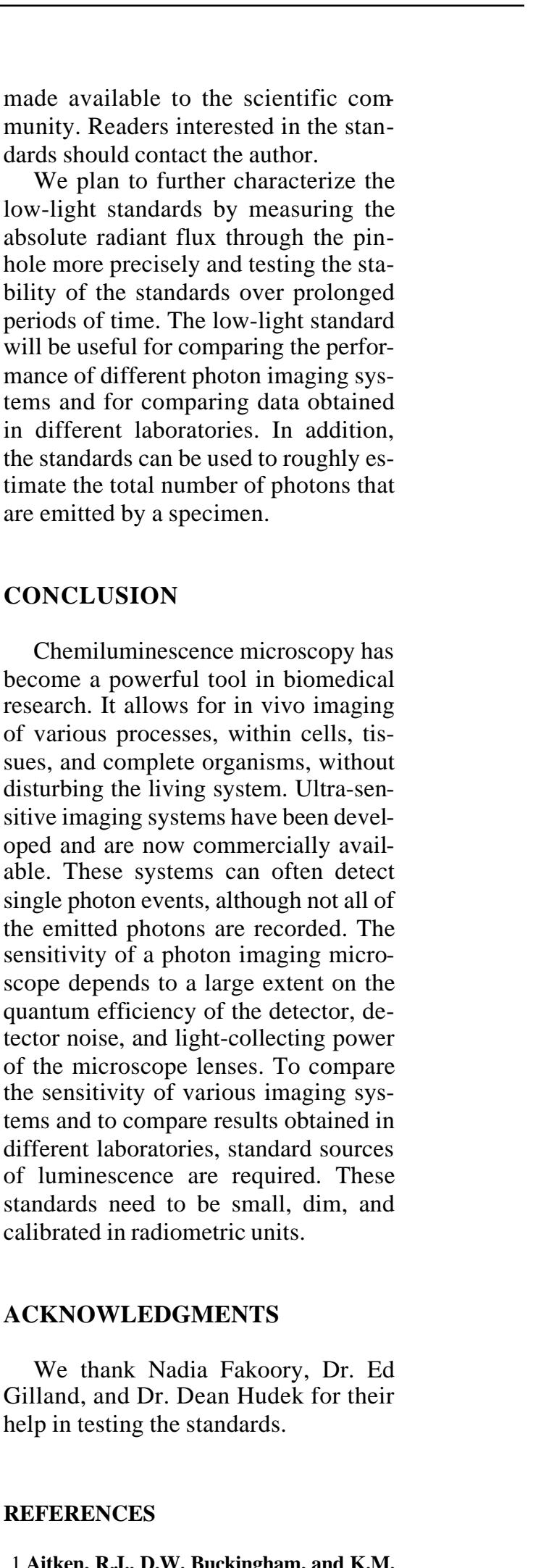

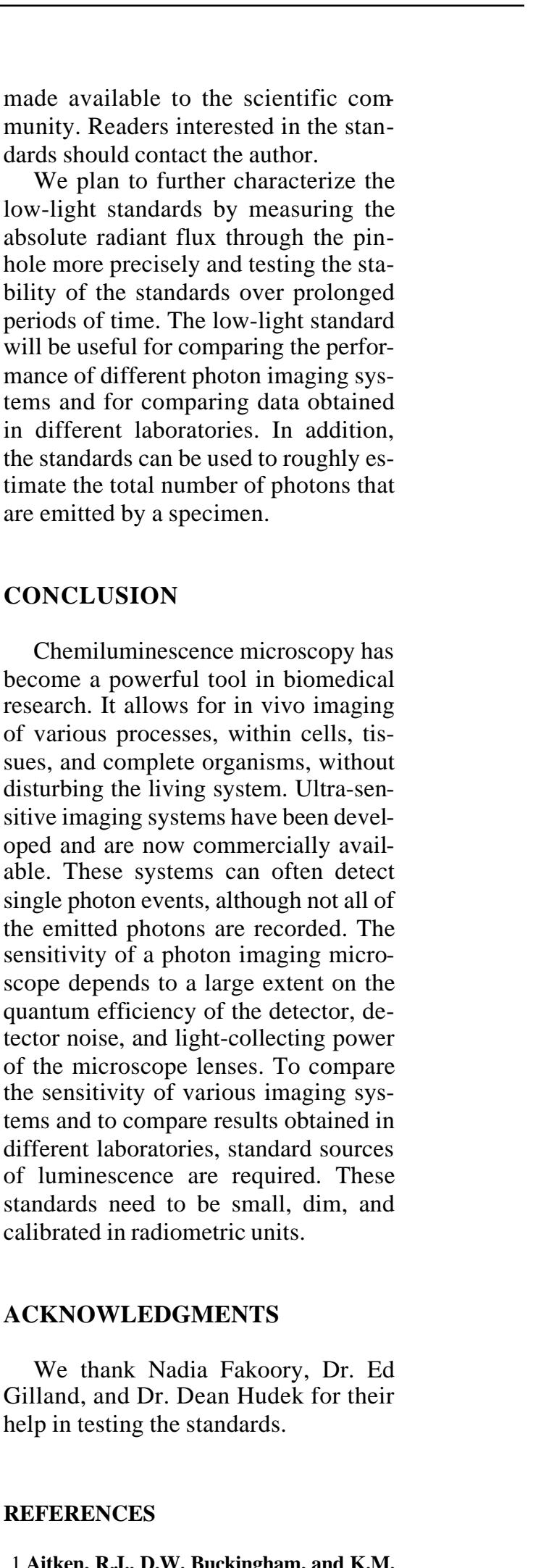

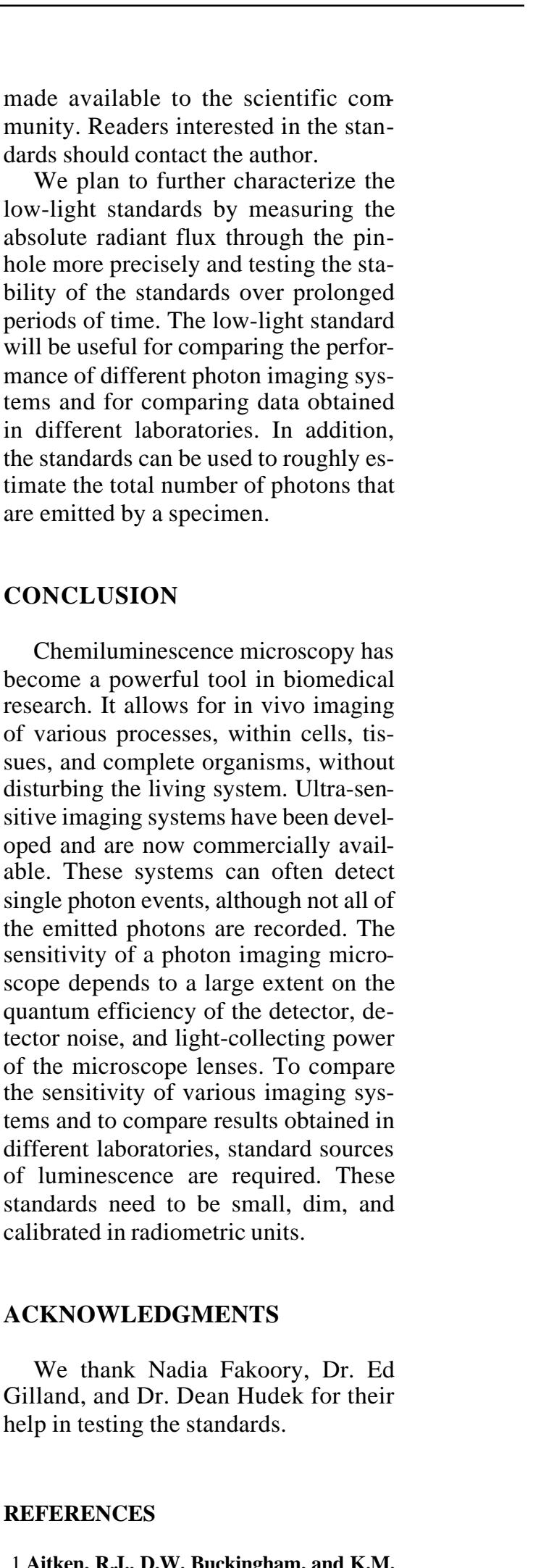

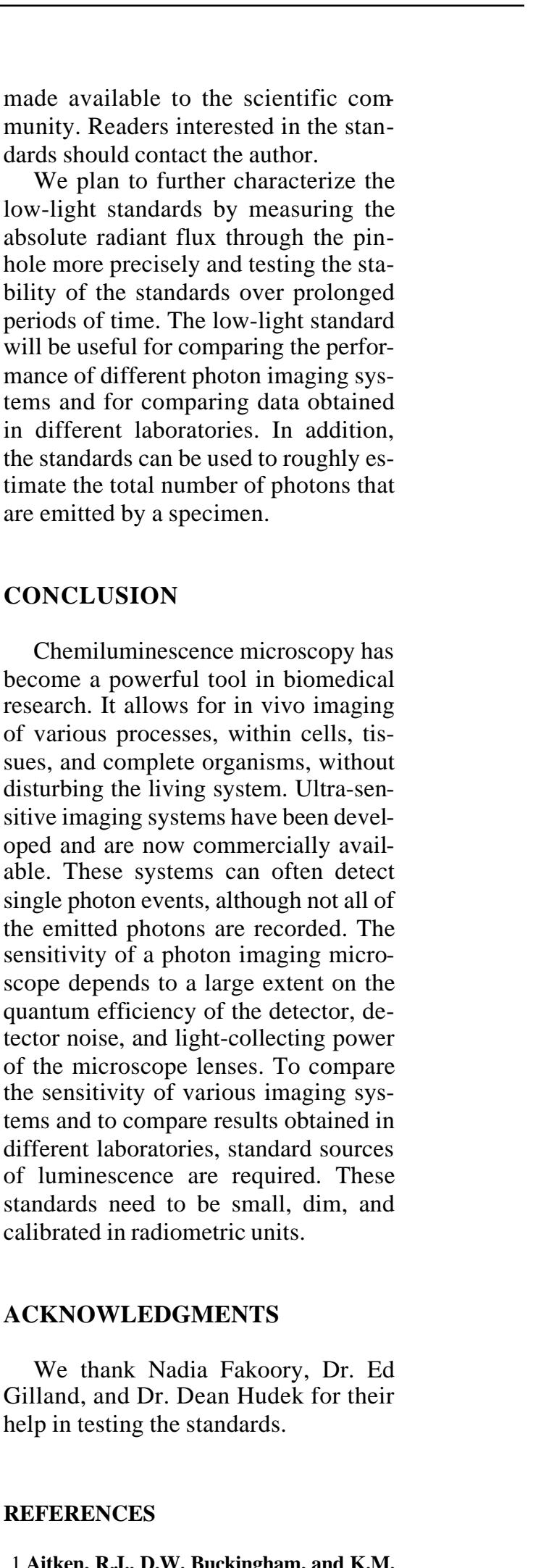

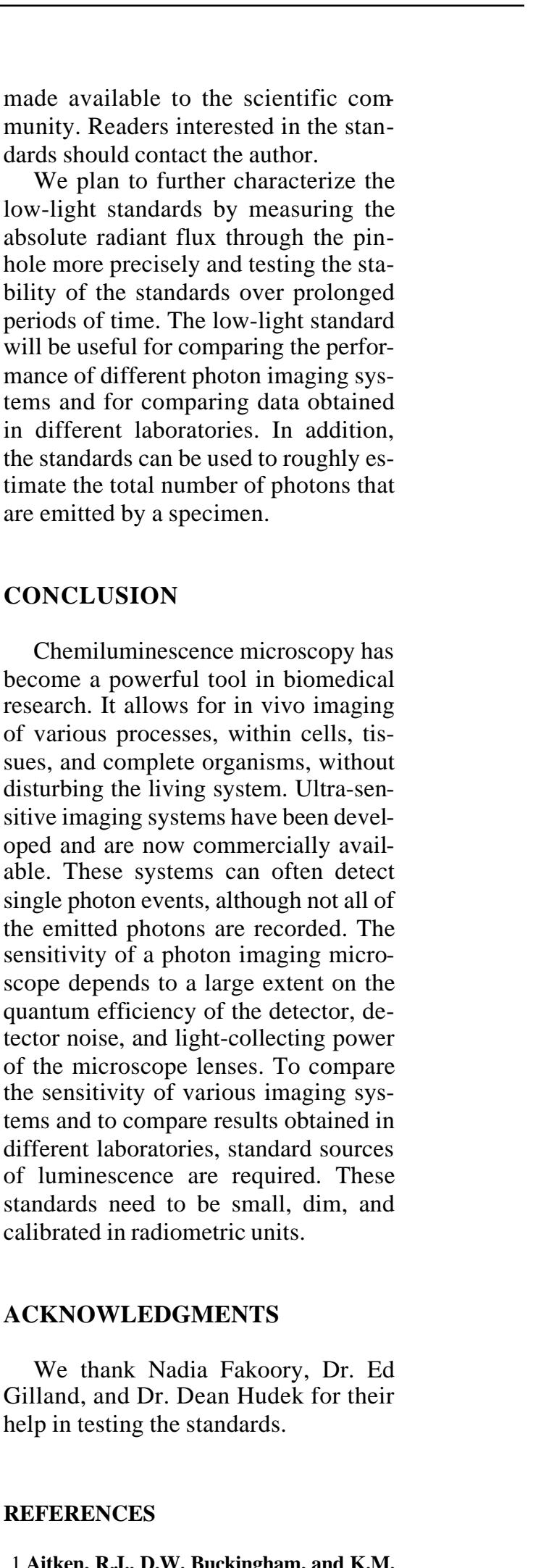

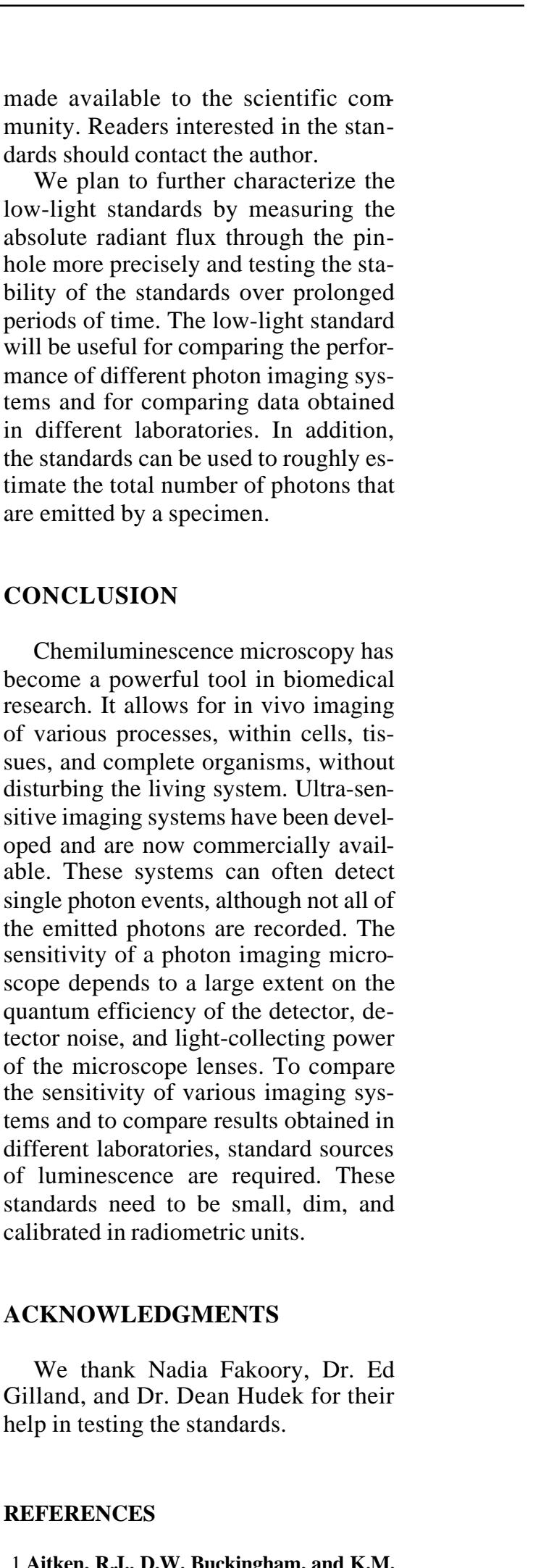

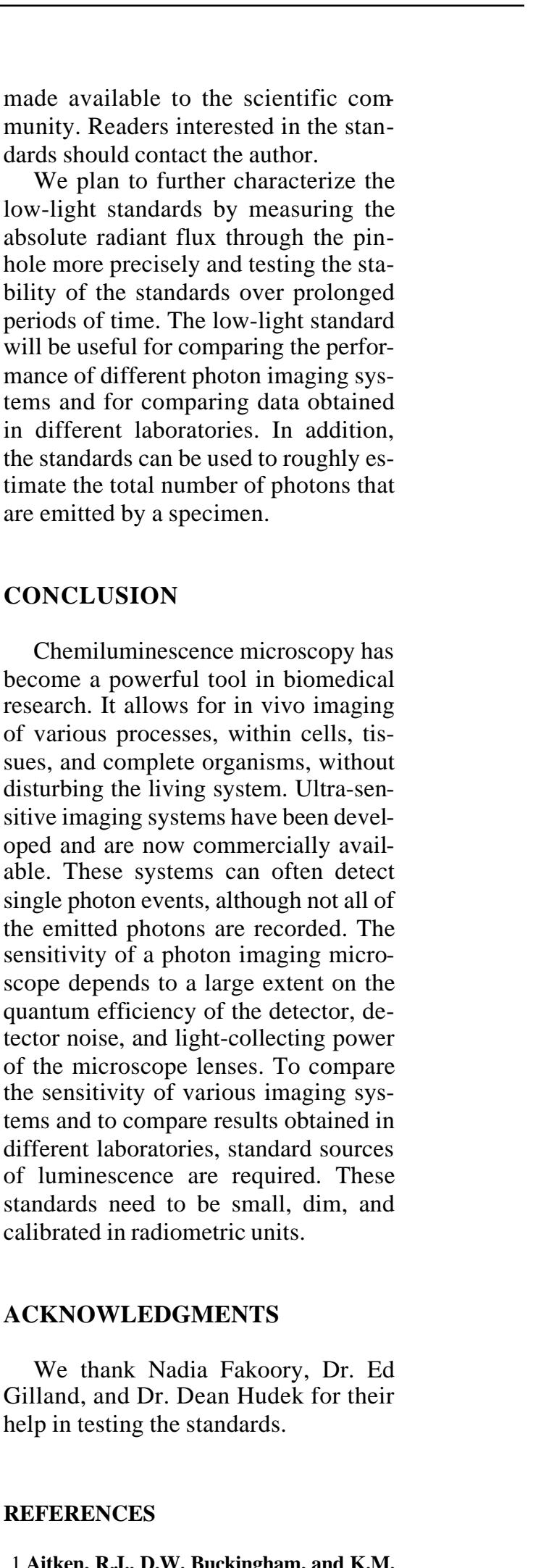

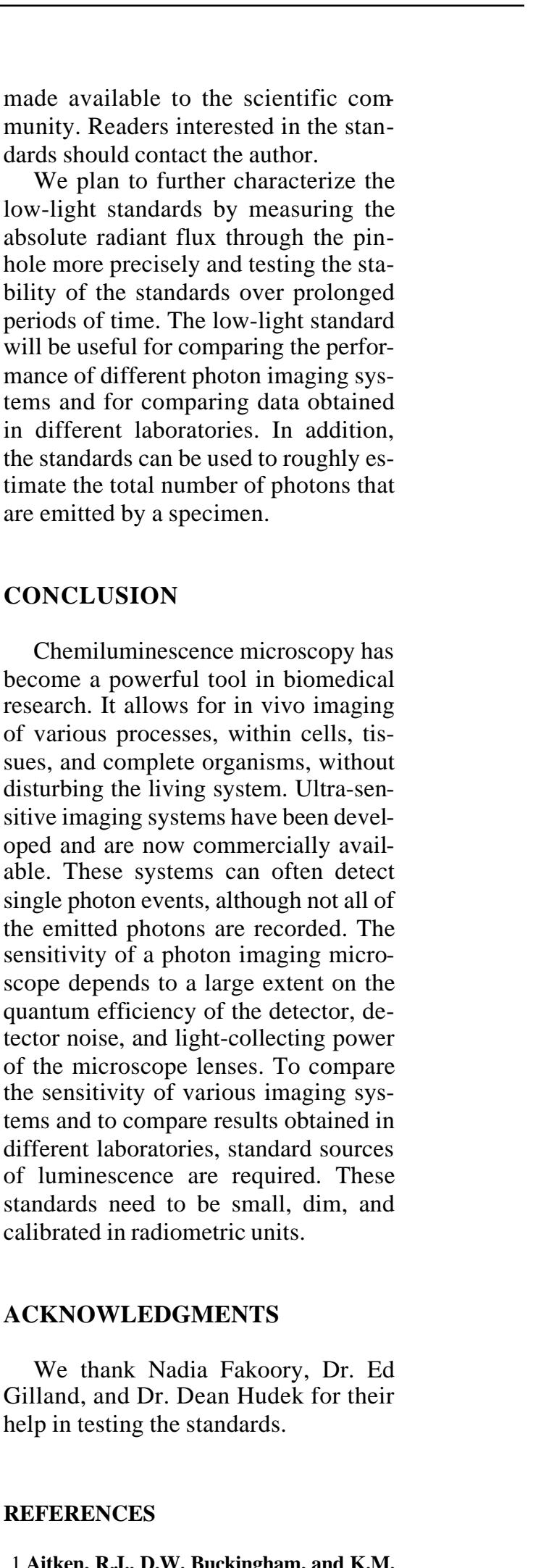

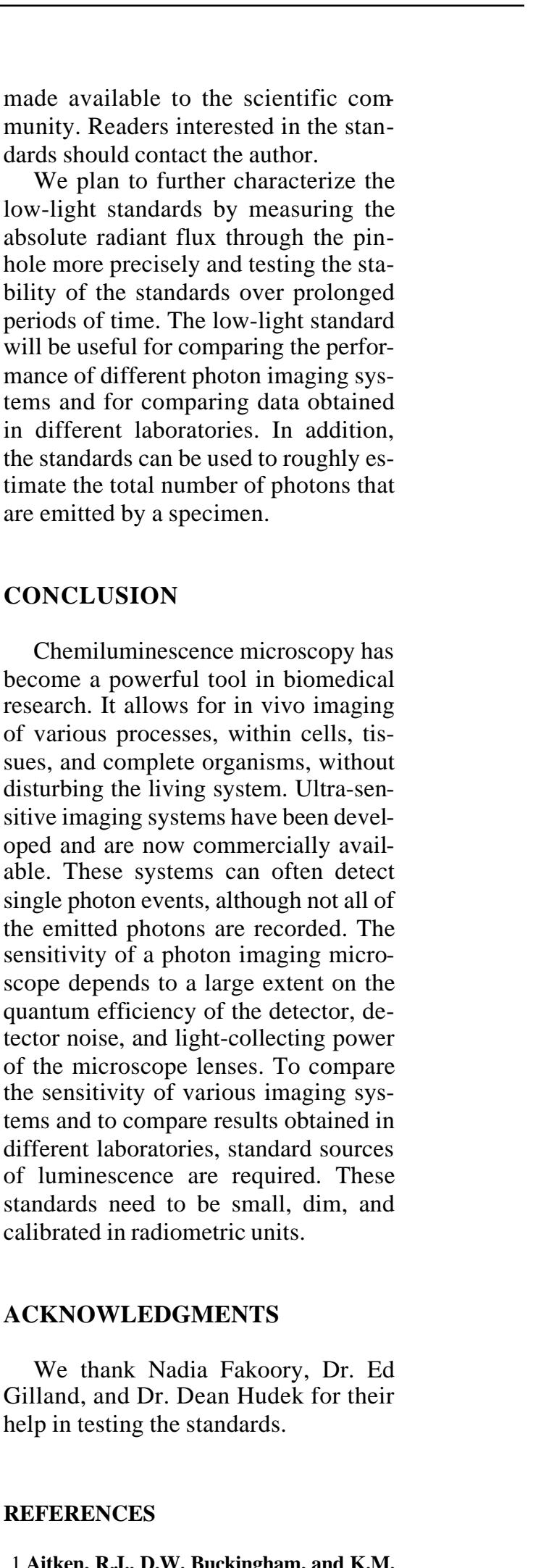

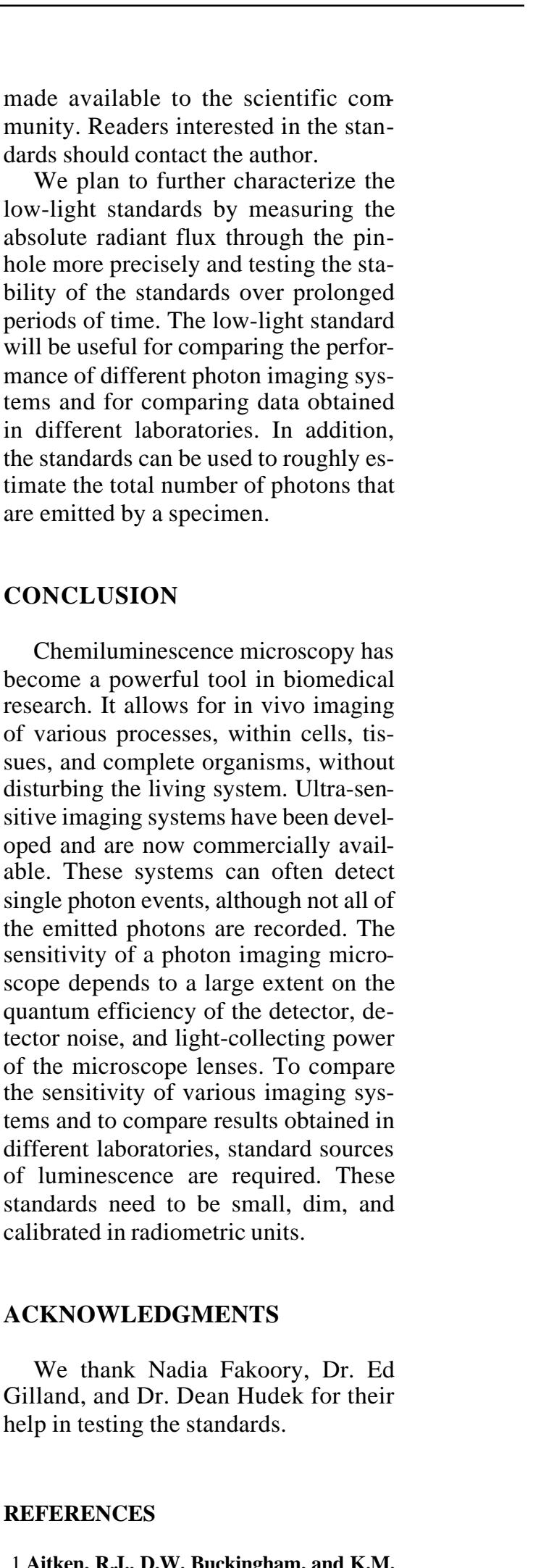

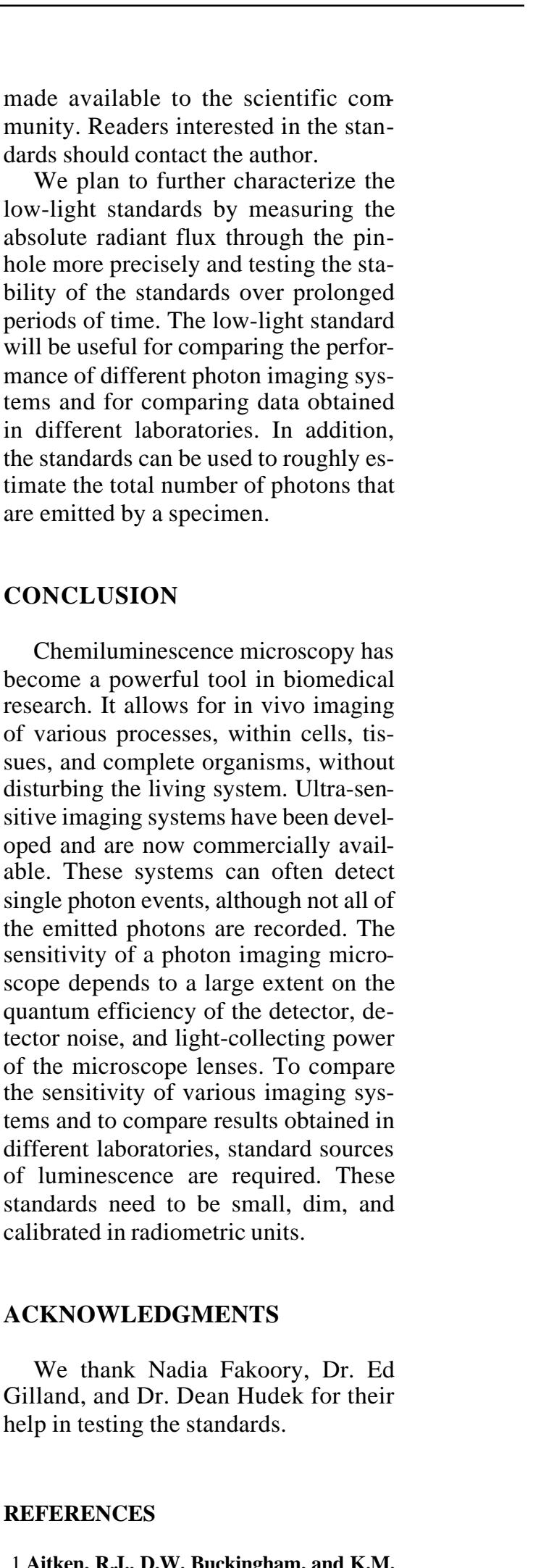

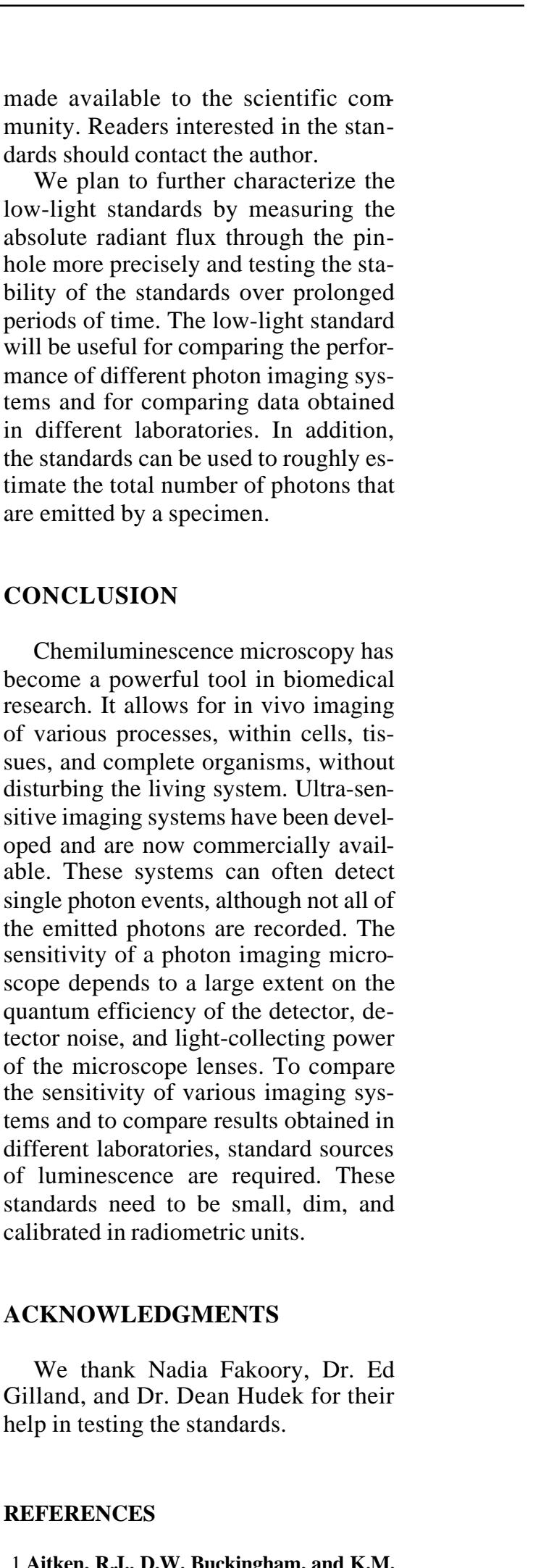

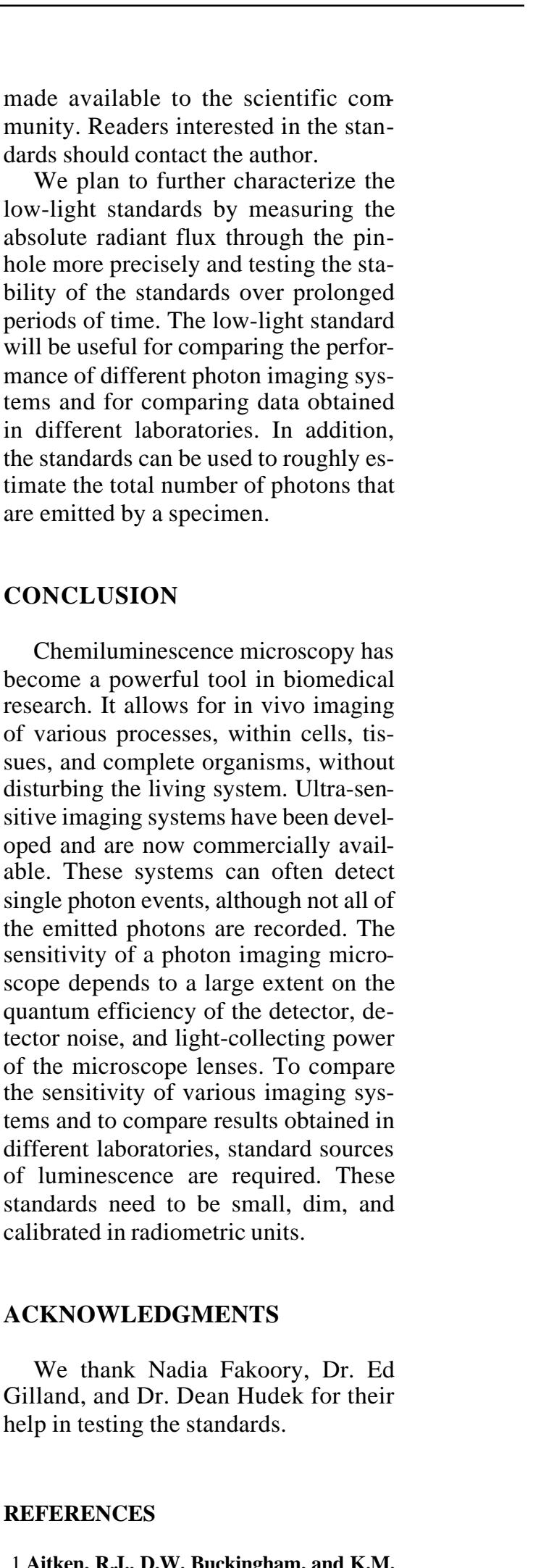

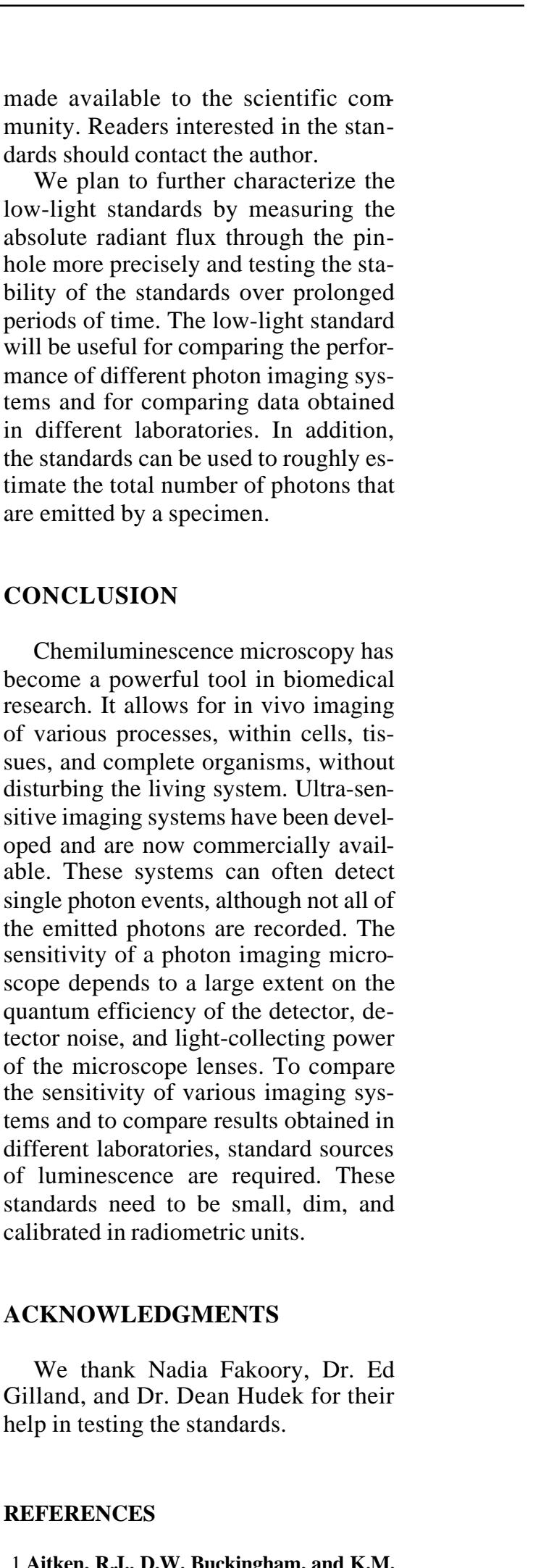

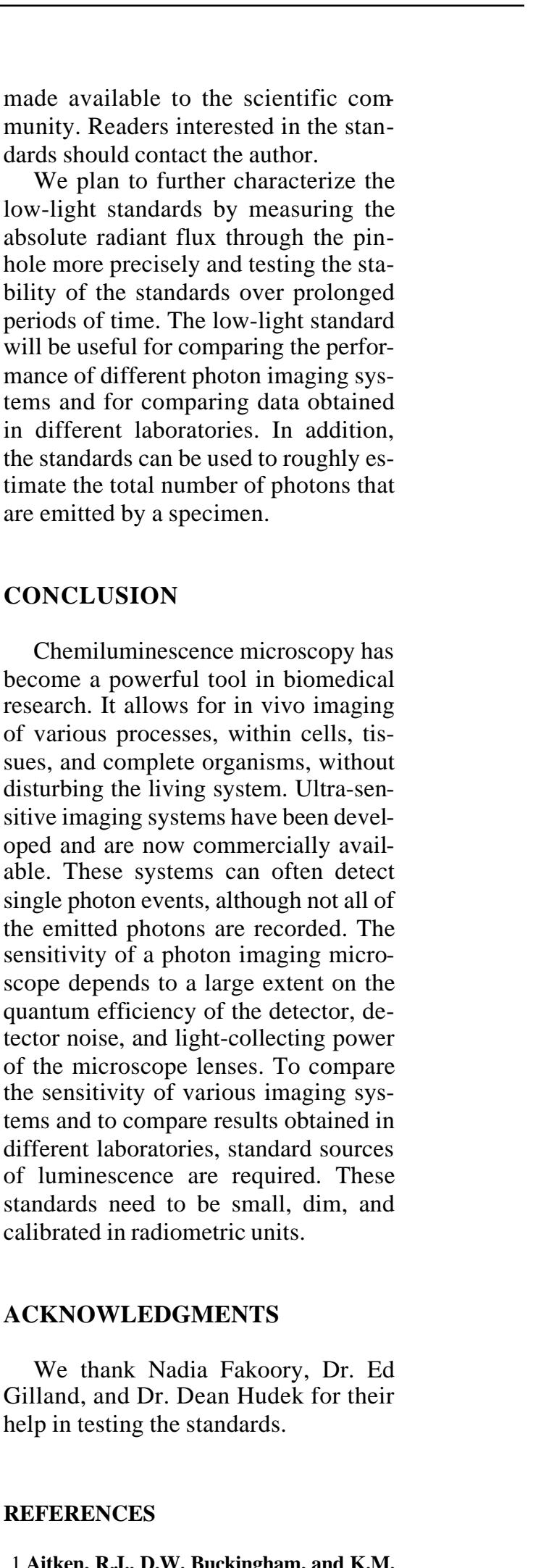

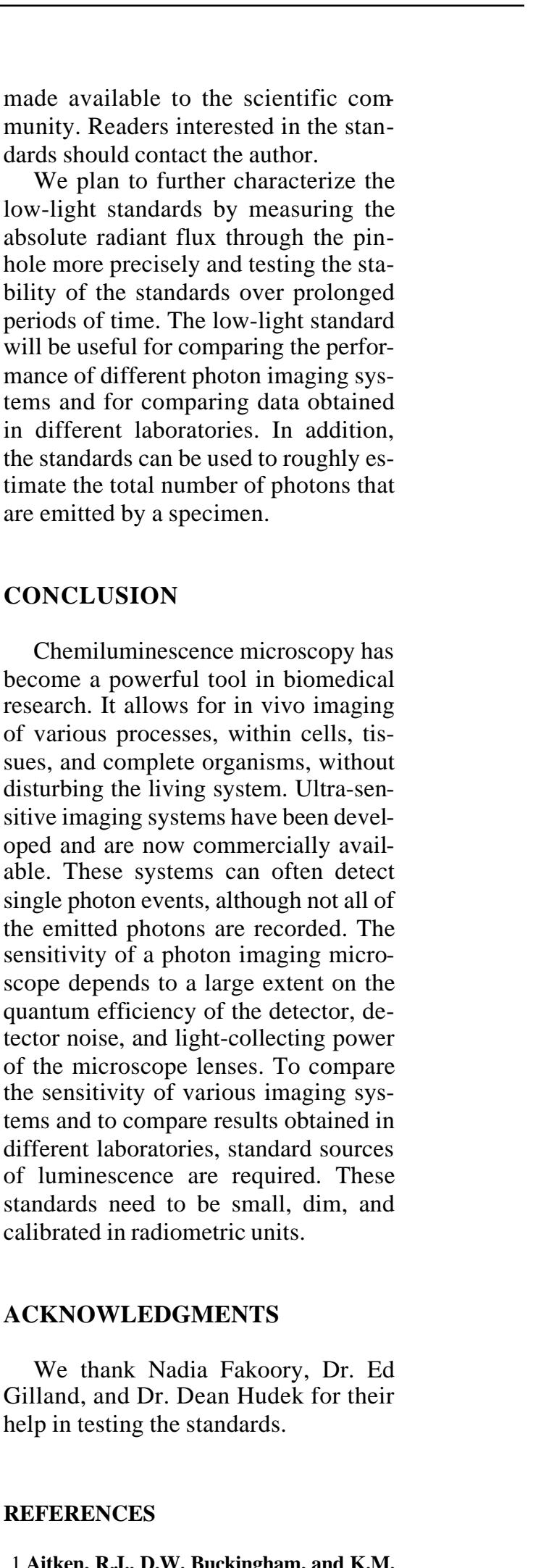

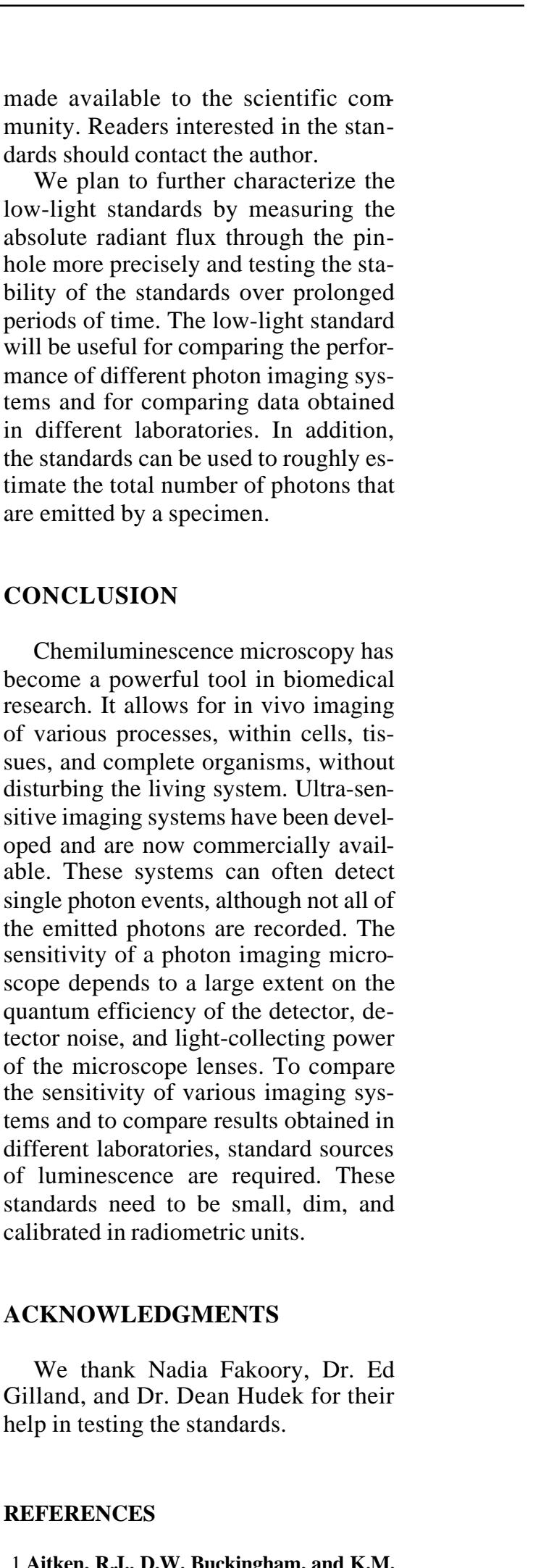

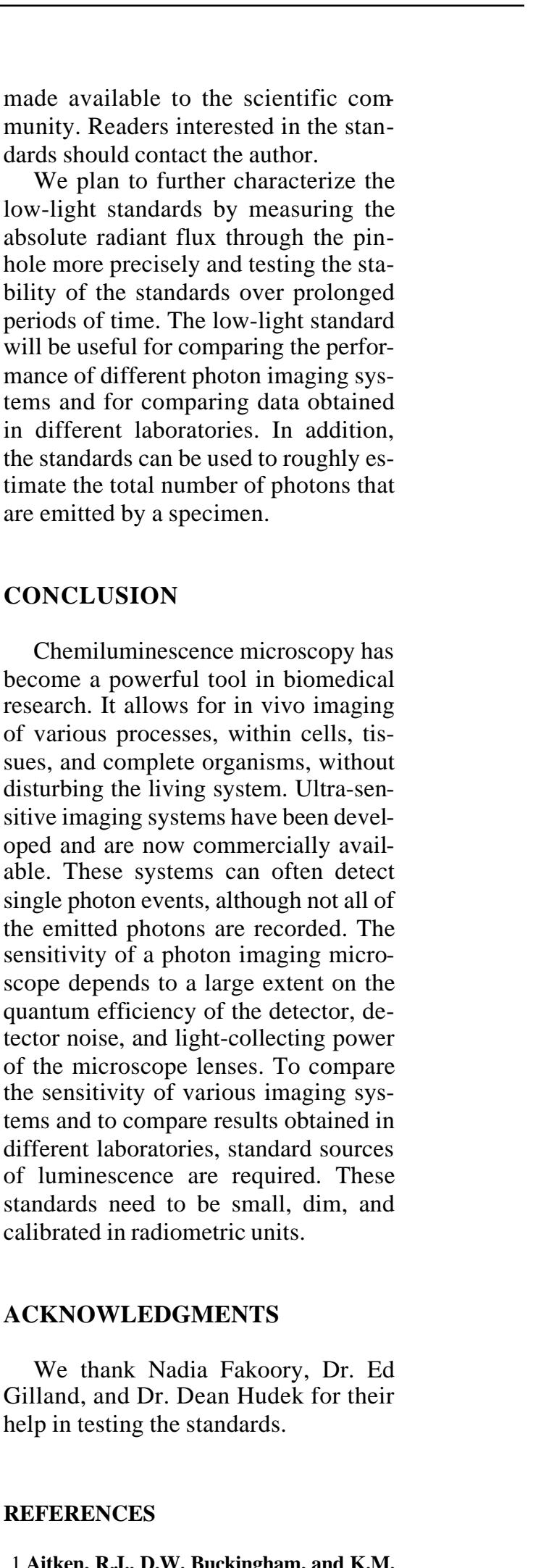

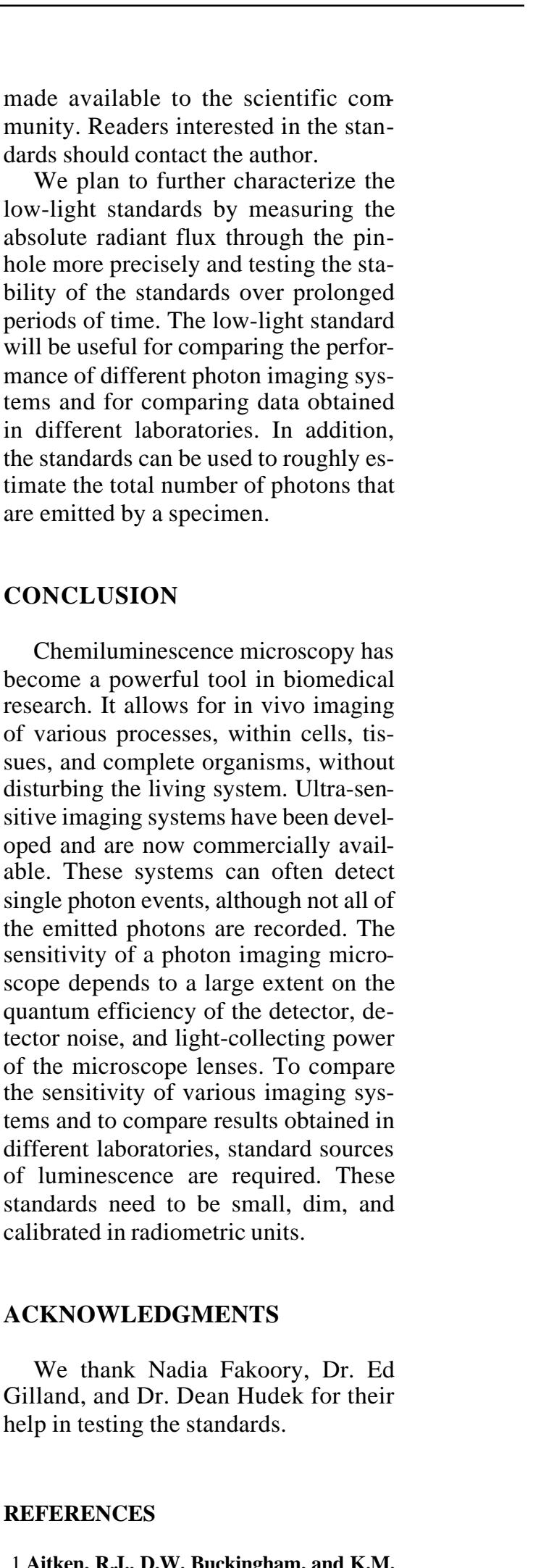

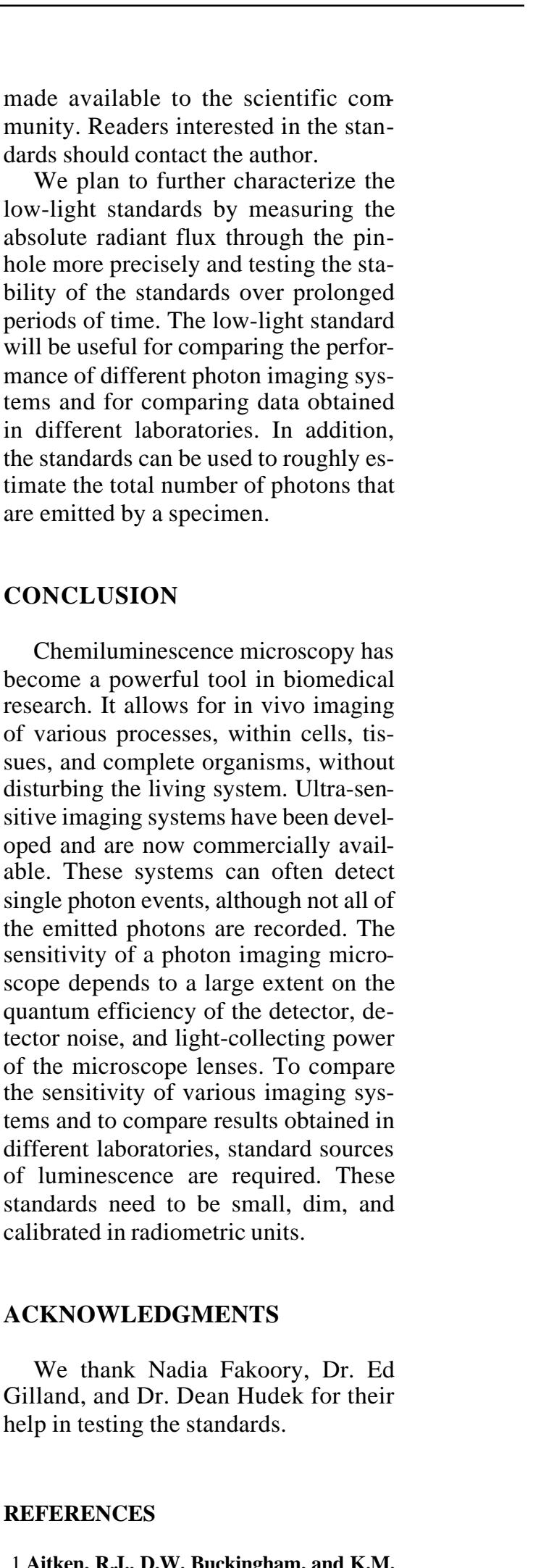

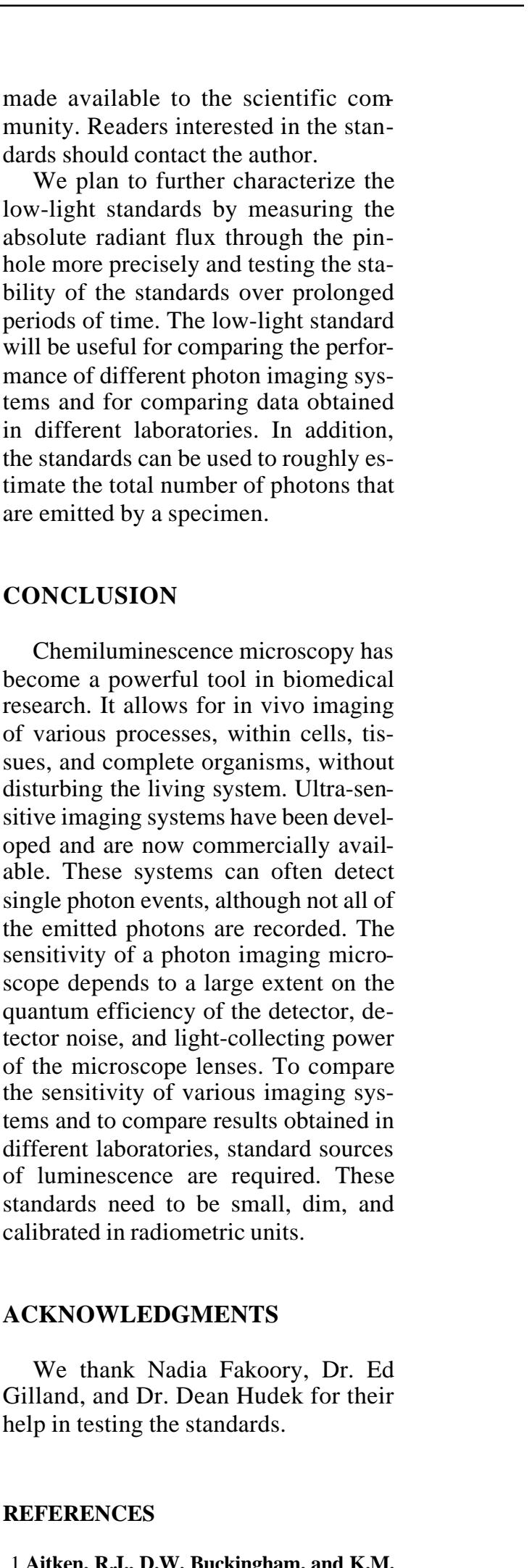

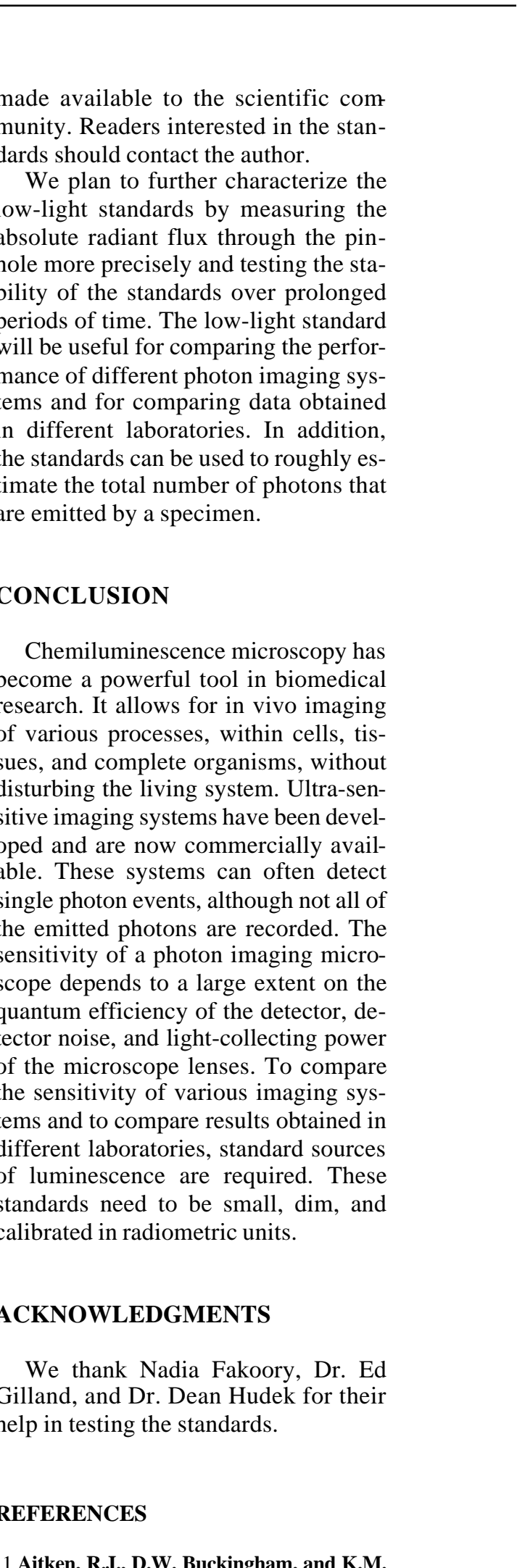

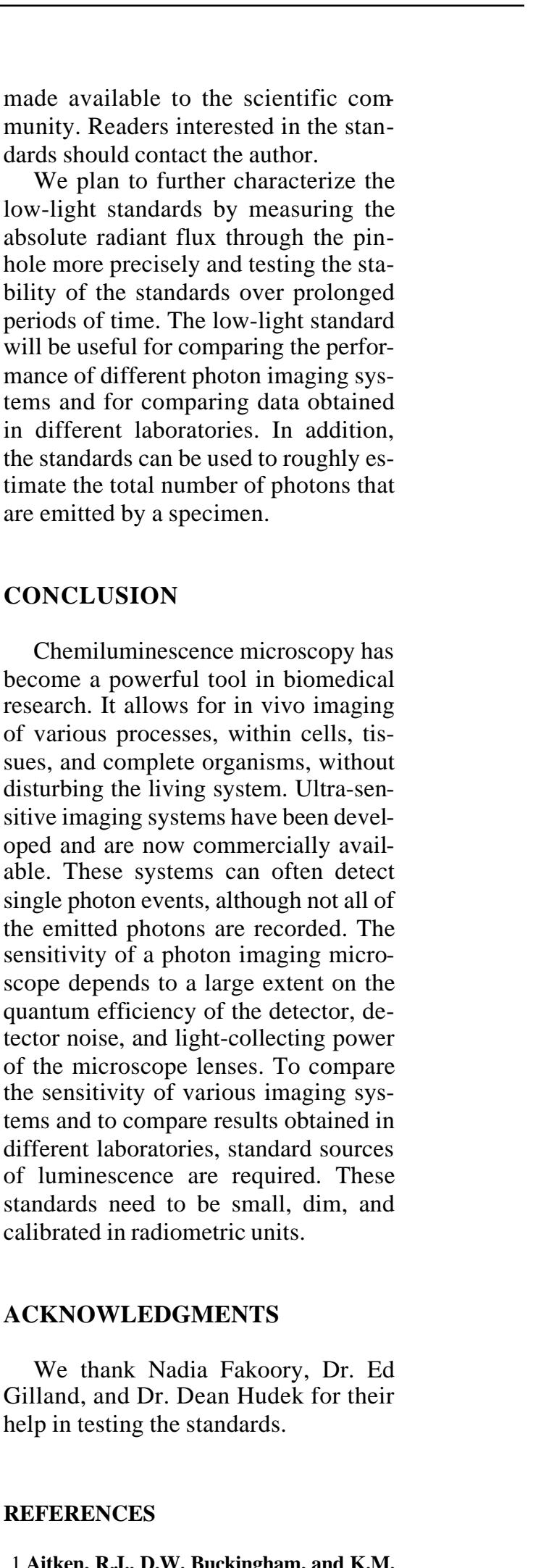

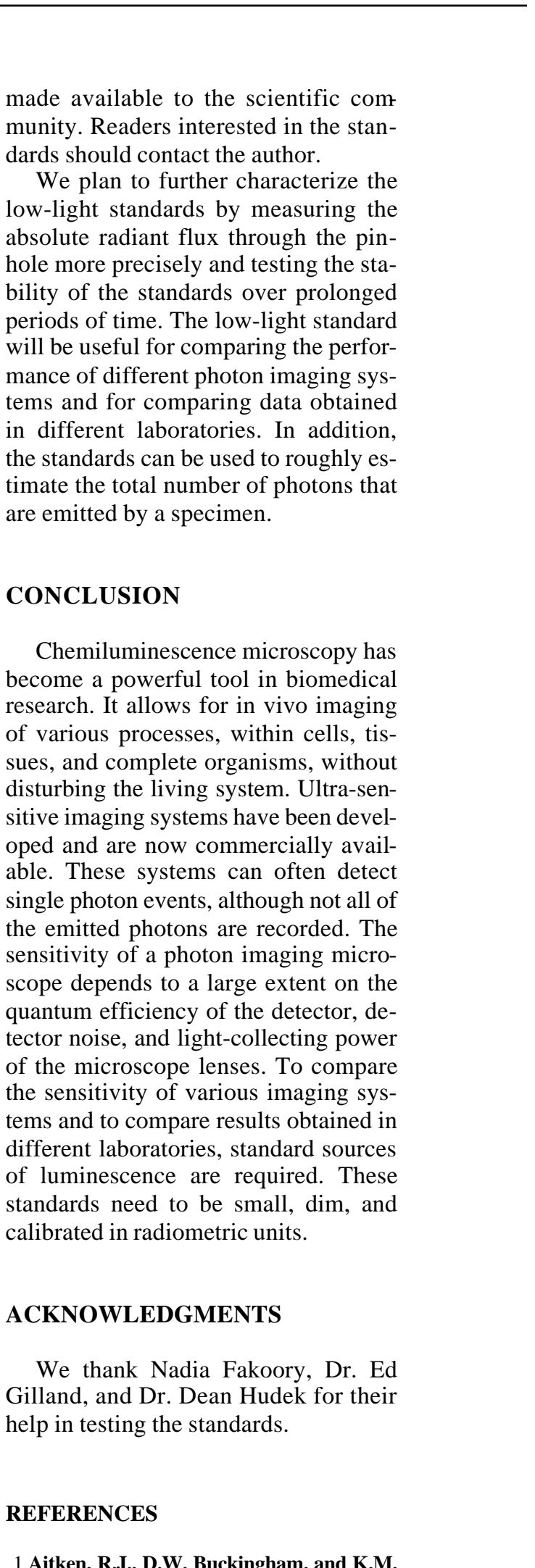

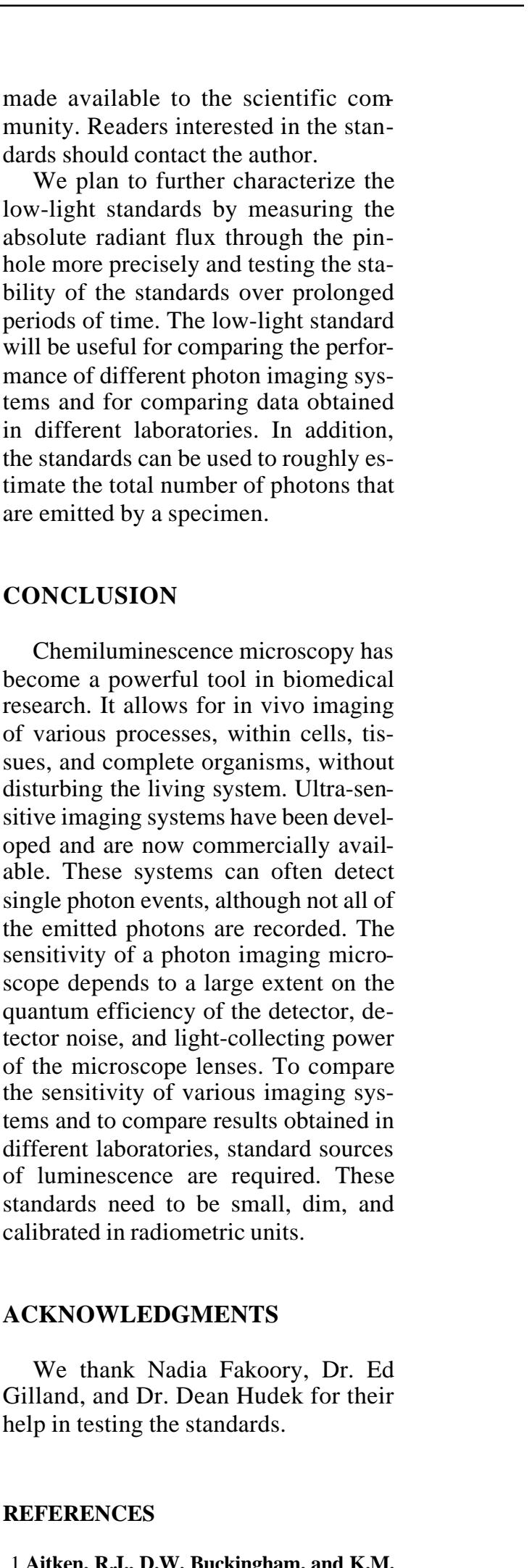

1.Aitken, R.J., D.W. Buckingham, and K.M. West. 1992. Reactive oxygen species and human spermatozoa: analysis of the cellular mechanisms involved in luminol- and lucigenin-dependent chemiluminescence. J. Cell. Physiol. 151:466-477.

2.Beach, J.M. 1997. A LED light calibration 
source for dual-wavelength microscopy. Cell Calcium 21:63-68.

3.Beach, J.M. and B.R. Duling. 1993. A lightemitting diode light standard for photo- and videomicroscopy. J. Microsc. 172:41-48.

4.Berthold, F., K. Herick, and R.M. Siewe. 2000. Luminometer design and low light detection. Methods Enzymol. 305:62-87.

5.Brini, M., P. Pinton, T. Pozzan, and R. Rizzuto. 1999. Targeted recombinant aequorins: tools for monitoring $\left[\mathrm{Ca}^{2+}\right]$ in the various compartments of a living cell. Microsc. Res. Tech. 46:380-389.

6.Bronstein, I., B. Edwards, and J.C. Voyta. 1989. 1,2-dioxetanes: novel chemiluminescent enzyme substrates. Applications to im munoassays. J. Biolumin. Chemilumin. 4:99111.

7.Créton, R., J.A. Kreiling, and L.F. Jaffe. 1999. Calcium imaging with chemiluminescence. Microsc. Res. Tech. 46:390-397.

8.Créton, R., J.A. Kreiling, and L.F. Jaffe. 2000. Presence and roles of calcium gradients along the dorsal-ventral axis in Drosophila embryos. Dev. Biol. 217:375-385.

9.Créton, R., J.E. Speksnijder, and L.F. Jaffe. 1998. Patterns of free calcium in zebrafish embryos. J. Cell Sci. 111:1613-1622.

10.Faulkner, K. and I. Fridovich. 1993. Luminol and lucigenin as detectors for $\mathrm{O}_{2}$. Free Radic. Biol. Med. 15:447-451.

11.Foerder, C.A., S.J. Klebanoff, and B.M. Shapiro. 1978. Hydrogen peroxide production, chemiluminescence, and the respiratory burst of fertilization: interrelated events in early sea urchin development. Proc. Natl. Acad. Sci. USA 75:3183-3187.

12.Hasan, M.T., K. Schonig, S. Berger, W. Graewe, and H. Bujard. 2001. Long-term, noninvasive imaging of regulated gene expression in living mice. Genesis 29:116-122.

13.Hooper, C.E., R.E. Ansorge, and J.G. Rushbrooke. 1994. Low-light imaging technology in the life sciences. J. Biolumin. Chemilumin. 9:113-122

14.Kobayashi, M., M. Takeda, T. Sato, Y. Yamazaki, K. Kaneko, K. Ito, H. Kato, and H. Inaba. 1999. In vivo imaging of spontaneous ultraweak photon emission from a rat's brain correlated with cerebral energy metabolism and oxidative stress. Neurosci. Res. 34:103113.

15.Kolar, C., E. Fejes, E. Adam, E. Schafer, S. Kay, and F. Nagy. 1998. Transcription of Arabidopsis and wheat $\mathrm{Cab}$ genes in single tobacco transgenic seedlings exhibits independent rhythms in a developmentally regulated fashion. Plant J. 13:563-569.

16.Kricka, L.J. 2000. Application of bioluminescence and chemiluminescence in biomedical sciences. Methods Enzymol. 305:333345.

17.Kricka, L.J., J.C. Voyta, and I. Bronstein. 2000. Chemiluminescent methods for detecting and quantitating enzyme activity. Methods Enzymol. 305:370-390.

18.Lilius, E.M. and P. Marnila. 1992. Photon emission of phagocytes in relation to stress and disease. Experientia 48:1082-1091.

19.Lundin, A. 2000. Use of firefly luciferase in ATP-related assays of biomass, enzymes, and metabolites. Methods Enzymol. 305:346-370.
20.Martin, C.S., P.A. Wight, A. Dobretsova, and I. Bronstein. 1996. Dual luminescencebased reporter gene assay for luciferase and $\beta$ galactosidase. BioTechniques 21:520-524

21.Matsumoto, K., M. Anzai, N. Nakagata, A. Takahashi, Y. Takahashi, and K. Miyata. 1994. Onset of paternal gene activation in early mouse embryos fertilized with transgenic mouse sperm. Mol. Reprod. Dev. 39:136-140.

22. McCapra, F. 2000. Chemical generation of excited states: the basis of chemiluminescence and bioluminescence. Methods Enzymol. 305:3-47.

23.McKinney, K.A., S.E. Lewis, and W. Thompson. 1996. Reactive oxygen species generation in human sperm: luminol and lucigenin chemiluminescence probes. Arch. Androl. 36:119-125.

24.Miller, A.L., E. Karplus, and L.F. Jaffe. 1994. Imaging $\left[\mathrm{Ca}^{2+}\right]$ with aequorin using a photon imaging detector. Methods Cell Biol. 40:305-338.

25.Mueller-Klieser, W. and S. Walenta. 1993. Geographical mapping of metabolites in biological tissue with quantitative bioluminescence and single photon imaging. Histochem. J. 25:407-420.

26.Nicolas, J.C. 1994. Applications of low-light imaging to life sciences. J. Biolumin. Chemilumin. 9:139-144.

27.Ochsendorf, F.R., J. Thiele, J. Fuchs, H. Schuttau, H.J. Freisleben, M. Buslau, and R. Milbradt. 1994. Chemiluminescence in semen of infertile men. Andrologia 26:289293.

28.O'Kane, D.J. and J. Lee. 2000. Absolute calibration of luminometers with low-level light standards. Methods Enzymol. 305:87-96.

29. Olesen, C.E., C.S. Martin, J. Mosier, B. Liu, J.C. Voyta, and I. Bronstein. 2000. Chemiluminescent reporter gene assays with 1,2-dioxetane enzyme substrates. Methods Enzymol. 305:428-450.

30.Plautz, J.D. and S.A. Kay. 1999. Synchronous real-time reporting of multiple cellular events. Methods Cell Biol. 58:283-291.

31.Rehemtulla, A., L.D. Stegman, S.J. Cardozo, S. Gupta, D.E. Hall, C.H. Contag, and B.D. Ross. 2000. Rapid and quantitative assessment of cancer treatment response using in vivo bioluminescence imaging. Neoplasia 2:491-495.

32.Roda, A., M. Musiani, P. Pasini, M. Baraldini, and J.E. Crabtree. 2000. In situ hybridization and immunohistochemistry with enzyme-triggered chemiluminescent probes. Methods Enzymol. 305:577-590.

33.Sala-Newby, G.B., M.N. Badminton, W.H. Evans, C.H. George, H.E. Jones, J.M. Kendall, A.R. Ribeiro, and A.K. Campbell. 2000. Targeted bioluminescent indicators in living cells. Methods Enzymol. 305:479-498.

34.Sala-Newby, G.B., K.M. Taylor, M.N. Badminton, C.M. Rembold, and A.K. Campbell. 1998. Imaging bioluminescent indicators shows $\mathrm{Ca}^{2}+$ and ATP permeability thresholds in live cells attacked by complement. Im munology 93:601-609.

35.Shimomura, O. 1995. A short story of aequorin. Biol. Bull. 189:1-5.

36.Stanley, P.E. 2000. Commercially available luminometers and low-level light imaging de- vices. Methods Enzymol. 305:96-103.

37.Stanley, P.E. 2000. Some brief notes on nomenclature and units and standards used in bioluminescence and chemiluminescence. Methods Enzymol. 305:47-50.

38. Takahashi, A., H. Totsune-Nakano, M. Nakano, S. Mashiko, N. Suzuki, C. Ohma, and H. Inaba. 1989. Generation of $\mathrm{O}_{2}$ - and tyrosine cation-mediated chemiluminescence during the fertilization of sea urchin eggs. FEBS Lett. 246:117-119.

39.Tamulevicius, P. and C. Streffer. 1995. Metabolic imaging in tumours by means of bioluminescence. Br. J. Cancer 72:1102-1112.

40.Teranishi, K. and O. Shimomura. 1997. Coelenterazine analogs as chemiluminescent probe for superoxide anion. Anal. Biochem. 249:37-43.

41.Van Wijk, R. and D.H. Schamhart. 1988. Regulatory aspects of low intensity photon emission. Experientia 44:586-593.

42.Voss, K.J. 2000. Physics of low light level detectors. Methods Enzymol. 305:53-61.

43.Wiklund, N.P., H.H. Iversen, A.M. Leone, S. Cellek, L. Brundin, L.E. Gustafsson, and S. Moncada. 1999. Visualization of nitric oxide formation in cell cultures and living tissue. Acta Physiol. Scand. 167:161-166.

44.Zalata, A., T. Hafez, and F. Comhaire. 1995. Evaluation of the role of reactive oxygen species in male infertility. Hum. Reprod. 10:1444-1451.

Address correspondence to:

Dr. Robbert Créton

Brown University School of Medicine

Dept. OB-GYN

Women \& Infants Hospital

101 Dudley Street

Providence, RI 02905, USA

e-mail: robbert_creton_phd@brown.edu

For reprints of this or any other article, contact Reprints@BioTechniques.com 